\title{
24. NEOGENE EXPLOSIVE VOLCANIC ACTIVITY OF THE WESTERN PACIFIC: SITES 292 AND 296, DSDP LEG 31
}

\author{
Thomas W. Donnelly, Department of Geological Sciences, State University \\ of New York, Binghamton, New York
}

\section{INTRODUCTION}

The examination of deep-sea sediments to elucidate terrestrial geological history continues to be one of the most important aspects of the Deep Sea Drilling Project. In particular, the examination of continuously cored sections near eruptive island-arc areas has proven to be the best means of determining the eruptive history of these arcs. Previous studies (Donnelly and Nalli, 1973; Donnelly, 1973) showed that the relatively primitive semiquantitative methods developed during Leg 15 could indicate the various periods of high and low-level volcanic activity in the Lesser Antilles. In relation to island-arc volcanic activity, the generation of calcalkaline magmas is generally believed to be the direct consequence of melting due to friction between a descending crustal slab beneath island arcs and the mantle above this slab. The rate of slab movement (or, at least, sea-floor spreading) is considered by many to be relatively constant, perhaps varying by a factor of two or less. Therefore, any large-scale variations in the generation of island-arc magmas with time would appear to be in contradiction to the notion of uniform spreading rates. The study of this variation with time should provide valuable insight into the question of uniformity or non-uniformity of spreading rate, or, at least, slab descent.

The western Pacific affords an excellent opportunity for further examination of the variation in volcanic activity. Sugimura and Uyeda (1973) have described the division of Japanese Neogene activity into two periods, exemplified by the classic occurrences around Hakone. Sugimura (personal communication) further modified the dates by placing a gap in activity between 13 m.y. and 2 m.y. (mid-middle Miocene through the Pliocene).

Samples from DSDP Site 296, on the Palau-Kyushu Ridge (about $500 \mathrm{~km}$ southeast of Kyushu Island), were examined to determine the eruptive history of this arc (Kuno, 1962). The site's location (Figure 1), makes it probable that most of the volcanic detritus is derived from southwest Japan and the Ryukyu Islands via the dominant west-to-east prevailing winds.

Kyushu-Ryukyu volcanic history may not be identical with that of east Japanese-Izu. For southwest Japan, Sugimura and Uyeda (1973) state that the northern zone had a Miocene history similar to east Japan. The median zone was active in the middle Miocene and again in the late Miocene, with activity ending in the early Pleistocene. The southern zone had important Miocene activity, at 14 m.y. and 21 m.y. The present analysis of samples from Site 296, which recorded pelagic sedimentation from the middle Miocene to Recent, partly sub- stantiates their chronology, but suggests important modifications.

Site 292 (Figure 2) is located about $400 \mathrm{~km}$ southeast of the presently active volcanic center of northern Luzon and $400 \mathrm{~km}$ east of the center in central-southern Luzon (Neumann van Padang, 1953). The volcanic history of Luzon is not well known, thus, the record from Site 292 should provide a major contribution toward its understanding. Unfortunately, data from this site probably cannot distinguish between the northern (Cagua, Babuyan Islands) and central-southern (Mayon-Taal) volcanic centers. However, the location of Mayon nearly south of Site 292 would seem to discriminate against the southern Luzon center.

\section{METHODS}

The basic sample preparation method used involved sieving the $>43 \mu$ fraction from 2 cc of sediment, acidifying in $0.1 N \mathrm{HCl}$ to remove calcareous fossils, and weighing the residue. The fraction of volcanic debris was estimated from slides studied under the microscope.

The accumulation rate of total volcanic material, and of plagioclase crystals, separately, is calculated using the estimated total sedimentation rates for the sediment. For this study, the shipboard paleontology was used, with the depth-time curves for Sites 292 and 296 being shown in Figures 3 and 4.

The validity of the method for estimating explosive activity, and, from this, igneous activity in general, depends on several assumptions. These are: (1) that the sample is representative of a wide time interval; (2) that the debris deposited is directly related to magma production; (3) that the debris surviving diagenesis is representative of that originally deposited; and (4) that the debris coarser than $43 \mu$ is representative of the original material produced. Some of these assumptions are difficult to defend a priori; however, the relative smoothness of the trends in activity with time and the crude correlation from area to area tend to support the method.

\section{Problems Inherent in the Estimations}

Diagenetic alteration may alter or eliminate some of the originally deposited volcanic materials, especially glass. For example, because of the relatively slow accumulation rates at Site 292, from about 35 to 110 meters (Figure 3), most of the glass was converted to phillipsite. The alteration of glass in the older, more rapidly accumulated, volcanic sediment (about 110 to $140 \mathrm{~m}$ ), apparently resulted from a long exposure to seawater during an early Miocene hiatus (at about 110 


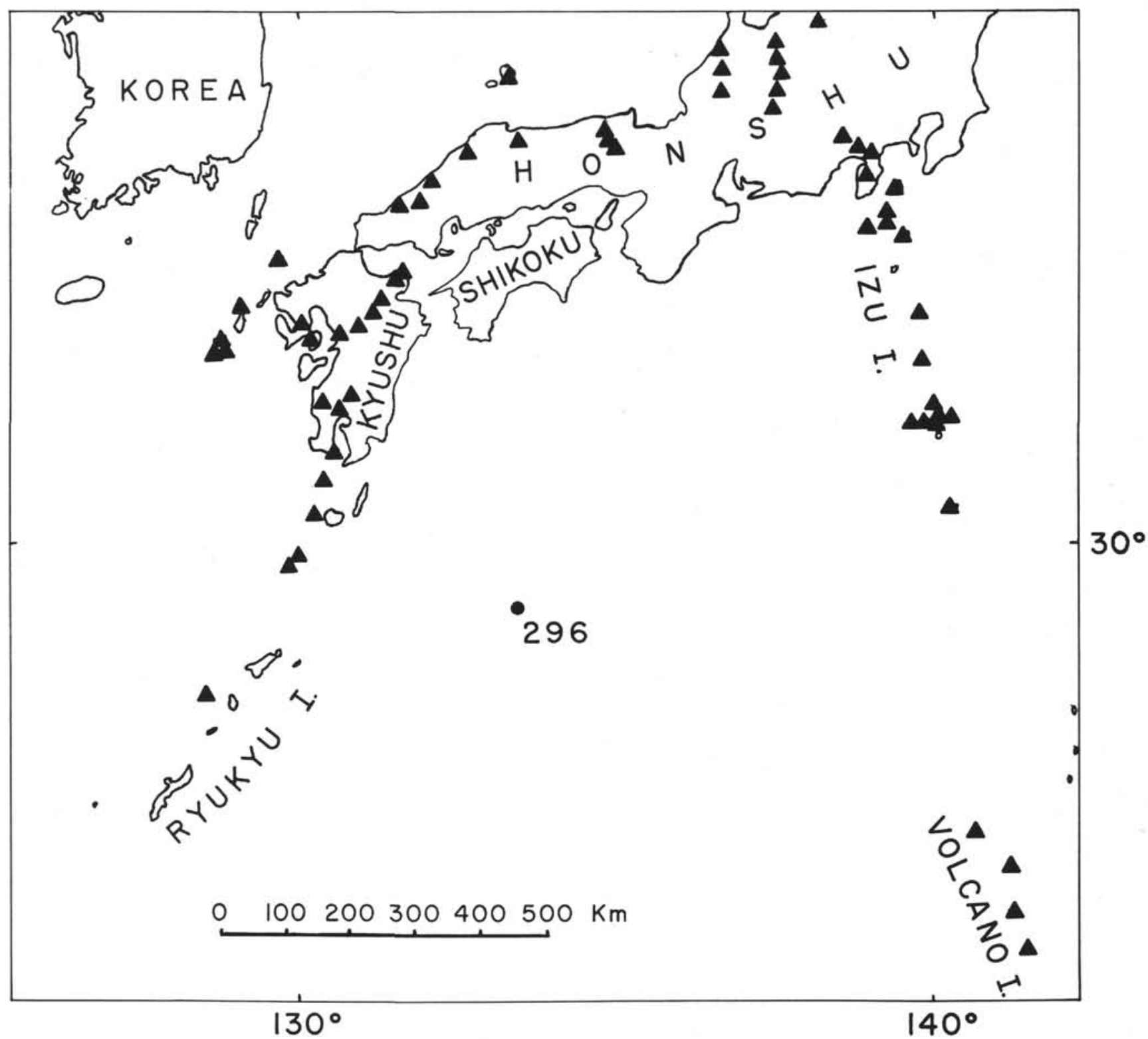

Figure 1. Location map showing for Site 296 Quaternary volcanic centers for Japan and adjacent areas (Kuno, 1962).

m). The absence of glass correlates well with the appearance of the phillipsite.

Thus an alternative method might be to estimate authigenic minerals instead of glass. However, the finegrained nature of the phillipsite, and the uncertainty on the authigenic origin of minerals, present difficulties which question the validity of this alternative estimation. Because of this, the principal determination is placed on the amount of plagioclase feldspar which, although sometimes corroded (Donnelly, 1973), is the most resistant abundant constituent of volcanic debris.

Questions are also raised as to whether the amount of plagioclase is reasonably constant in volcanic debris during periods of high and low eruptive activity. Evidence from Site 296 (Table 1) shows that glass is relatively more abundant during periods of high eruptive ac- tivity. Thus, estimates of variations in volcanic activity based on plagioclase alone might be underestimated.

Even when glass is fairly well preserved, not all volcanic debris is recognizable. The porphyritic andesitic glass from Sites 292 and 296 is commonly altered and nearly indistinguishable from fine-grained authigenic mineral aggregates.

Plagioclase, quartz, and K-feldspar of volcanic origin are not always easily distinguished from terrigenous debris deposited by turbidity currents. For example, quartz is found associated with epidote at Site 292, thus implying that the sediments might be sedimentary detritus with a low-grade metamorphic provenance.

The problem of $\mathrm{K}$-feldspar is more complex. Authigenic $\mathrm{K}$-feldspar, which is abundant in the Caribbean (Donnelly and Nalli, 1973), is even more abundant 


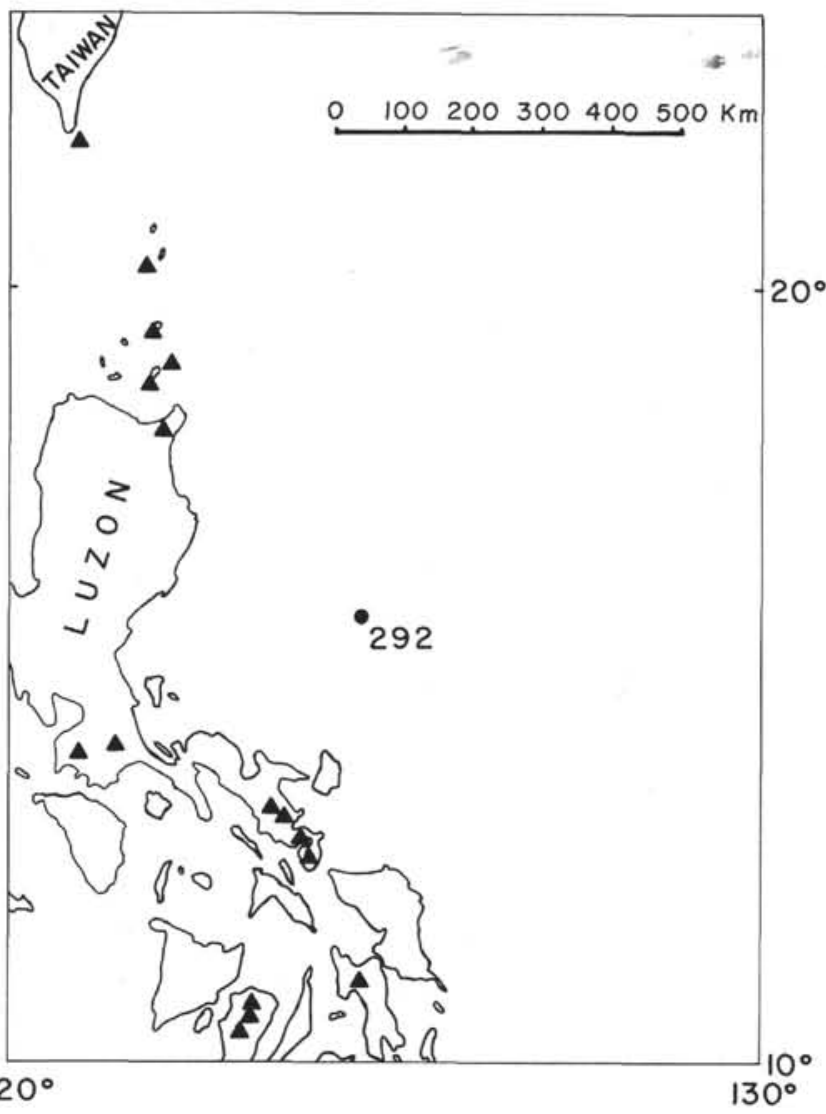

Figure 2. Location map showing Site 292 and Recent volcanic centers for the northern Philippines and adjacent areas (Neumann van Padang, 1956).

in the sediments of Sites 292 and 296. However, the grains are less euhedral than in the Caribbean occurrences and the distinction from volcanic K-feldspar is difficult. However, the results are not strongly affected by a misallocation of the feldspar types.

\section{Constituents and Calculations}

Microscopically, the following constituents are distinguished: clear, porphyritic, and brown glass; plagioclase; K-feldspar (volcanic versus authigenic distinguished); quartz; red, green, or brown hornblende; orthopyroxene; clinopyroxene; biotite; zircon; and apatite. The percentage of total volcanic material, clear glass, plagioclase, and "mafics" are found in Table 1 and illustrated on Plates 1-4. These constituents and some additional ones seen in samples from Sites 293, 294, and 295 are tabulated in Table 2.

Figures 5 and 6 show the logarithm of the rate of accumulation of total volcanic material, and of plagioclase alone, plotted against age. The rate of accumulation is calculated as follows: rate of accumulation $\left(\mathrm{g} / \mathrm{cm}^{2} \mathrm{~m} . \mathrm{y}\right.$.) $=w t$ of residue from $2 \mathrm{cc}$ of sediment $(\mathrm{g}) \times$ total sedimentation rate $(\mathrm{m} . / \mathrm{m} . \mathrm{y}) \times$.$50 \times fraction total$ volcanic material (or plagioclase).

It should be stressed that, although the method is rapid, the results are largely based on estimates of constituent abundance.

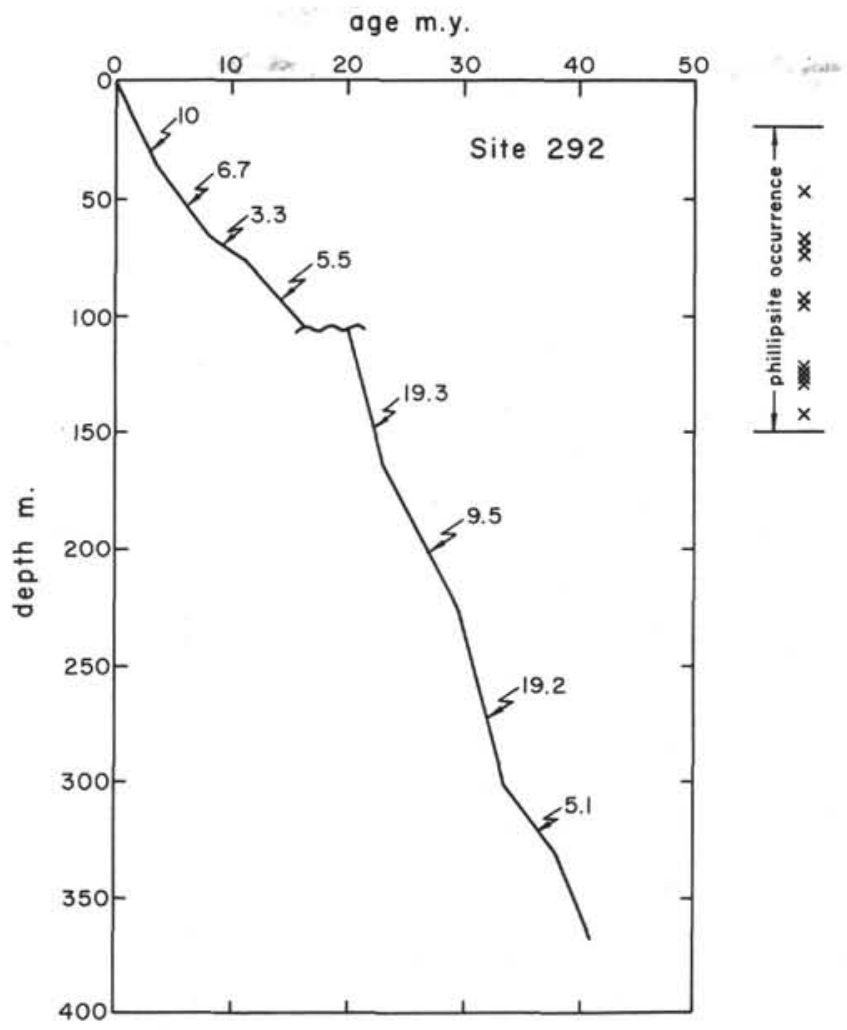

Figure 3. Age-depth curve for Site 292, showing total sediment accumulation rates used for construction of Figure 7.

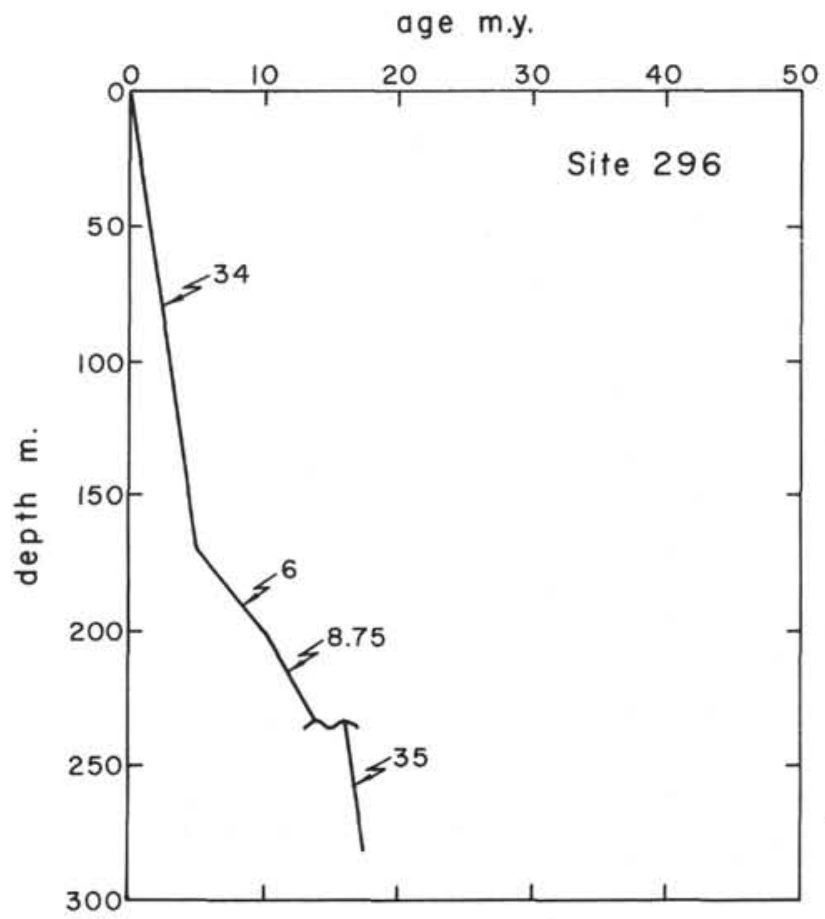

Figure 4. Age-depth curve for Site 296, showing total sediment accumulation rates used for construction of Figure 8. 
TABLE 1

Constituents Distinguished From Samples at Sites 292 and 296

\begin{tabular}{|c|c|c|c|c|c|c|c|c|c|c|}
\hline $\begin{array}{l}\text { Sample (Top of } \\
\text { Interval in } \mathrm{cm} \text { ) }\end{array}$ & Age & Wt Res & $\begin{array}{l}\text { Volc } \\
(\%)\end{array}$ & $\begin{array}{l}\text { Glass } \\
(\%)\end{array}$ & $\begin{array}{r}\text { Plag } \\
(\%)\end{array}$ & $\begin{array}{l}\text { Maf } \\
(\%)\end{array}$ & $\mathrm{Hb}$ & Orpx & Clpx & Other \\
\hline \multicolumn{11}{|l|}{ Site 292} \\
\hline $1-1,74$ & 0.09 & 0.025 & 60 & 30 & 29 & 10 & 45 & 14 & 42 & Q, Bi, red $\mathrm{Hb}$ \\
\hline $1-2,90$ & 0.29 & 0.331 & 90 & 45 & 18 & 2 & $(0)$ & (0) & $(100)$ & \\
\hline $1-3,85$ & 0.47 & 0.184 & 95 & 65 & 23 & 5 & 92 & 8 & 0 & red $\mathrm{Hb}$ \\
\hline $1-4,7$ & 0.55 & 0.03 & 95 & 50 & 35 & 16 & 100 & $\operatorname{tr}$ & $\operatorname{tr}$ & red $\mathrm{Hb}$ \\
\hline $2-1,143$ & 0.96 & 0.05 & 80 & 5 & 68 & 17 & 100 & $\operatorname{tr}$ & $\operatorname{tr}$ & $\mathrm{Bi}$, red $\mathrm{Hb}, \mathrm{KF}$ \\
\hline $2-2,66$ & 1.05 & 0.017 & 20 & 5 & 90 & 5 & 48 & 21 & 30 & $\mathrm{KF}$, red $\mathrm{Hb}, \mathrm{AKF}$ \\
\hline $2-3,91$ & 1.26 & 0.1055 & 90 & 70 & 20 & 5 & 16 & 16 & 68 & $\mathrm{KF}$ \\
\hline $3-1,148$ & 2.12 & 0.08 & 40 & 80 & 15 & 5 & 7 & 0 & 93 & $\mathrm{Bi}, \mathrm{Ep}$ \\
\hline $3-2,15 \mathrm{~A}$ & 2.14 & 0.6255 & 95 & 65 & 14 & 1 & 25 & 0 & 75 & $\mathrm{Bi}, \mathrm{Ep}$ \\
\hline $3-2,112$ & 2.26 & 0.018 & 30 & $\operatorname{tr}$ & 40 & 4 & 31 & 0 & 69 & $\mathrm{Bi}, \mathrm{Q}, \mathrm{AKF} *, \mathrm{P}, \mathrm{Ep}$ \\
\hline $3-3,17$ & 2.32 & 0.016 & 80 & $\operatorname{tr}$ & 85 & 15 & 72 & 2 & 27 & $\mathrm{Bi}, \mathrm{Ep}, \mathrm{P}, \mathrm{Z}$ \\
\hline $3-4,30$ & 2.52 & 0.003 & 90 & 0 & 87 & 13 & 71 & 0 & 29 & $\mathrm{Q}, \mathrm{Ep}, \mathrm{AKF}, \mathrm{P}$ \\
\hline $4-1,91$ & 3.20 & 0.003 & 85 & $\operatorname{tr}$ & 90 & 10 & 93 & 1 & 6 & red $\mathrm{Hb}^{*}, \mathrm{Ep}, \mathrm{P}$ \\
\hline $4-2,65$ & 3.35 & 0.005 & 70 & 1 & 81 & 10 & 94 & 0 & 6 & $\mathrm{Bi}$, red $\mathrm{Hb}, \mathrm{Q}, \mathrm{Ep}, \mathrm{P}$ \\
\hline $4-3,62$ & 3.53 & 0.002 & 30 & 1 & 96 & 2 & 76 & 0 & 24 & $\mathrm{Bi}$, red $\mathrm{Hb}, \mathrm{Ep}, \mathrm{P}, \mathrm{AKF}$ \\
\hline $4-4,127$ & 3.79 & 0.003 & 90 & $\operatorname{tr}$ & 83 & 12 & 83 & 0 & 17 & Ep \\
\hline $4-5,103$ & 3.94 & 0.002 & 20 & 0 & 70 & 20 & 64 & 0 & 36 & $\mathrm{Bi}$, red $\mathrm{Hb}, \mathrm{KF}^{*}, \mathrm{P}$ \\
\hline $5-1,22$ & 4.27 & 0.0012 & 40 & 1 & 94 & 2 & 69 & 2 & 29 & red $\mathrm{Hb}, \mathrm{Q}, \mathrm{Ep}^{*}, \mathrm{AKF}^{*}$ \\
\hline $5-2,74$ & 4.51 & 0.001 & 30 & 0 & 80 & 20 & 65 & 0 & 35 & $\mathrm{Bi}$, red $\mathrm{Hb}, \mathrm{Ep}, \mathrm{AKF}, \mathrm{Z}, \mathrm{P}$ \\
\hline $5-3,63$ & 4.68 & 0.0015 & 20 & 0 & 83 & 20 & 41 & 0 & 59 & $\mathrm{Ep}, \mathrm{AKF}$ \\
\hline $5-4,45$ & 4.84 & 0.0012 & 70 & 3 & 87 & 5 & 88 & 0 & 12 & $\mathrm{Ep}, \mathrm{AKF}, \mathrm{Z}$ \\
\hline $5-5,24$ & 5.00 & 0.0015 & 50 & 0 & 89 & 5 & 76 & 0 & 24 & $\mathrm{Q}, \mathrm{Zr}, \mathrm{Ep}, \mathrm{AKF}$ \\
\hline $5-6,60$ & 5.22 & $\operatorname{tr}$ & 40 & 1 & 89 & 10 & 89 & 0 & 11 & $\mathrm{Bi}$, red $\mathrm{Hb}, \mathrm{AKF}, \mathrm{P}$ \\
\hline $6-1,128$ & 5.55 & $\operatorname{tr}$ & 10 & 2 & 83 & 15 & 74 & 0 & 26 & red $\mathrm{Hb}, \mathrm{Ep}, \mathrm{AKF}, \mathrm{P}$ \\
\hline $6-2,35$ & 5.62 & 0.008 & 95 & 3 & 92 & 5 & 91 & 0 & 9 & $\mathrm{P}^{*}$ \\
\hline $6-3,68$ & 5.84 & 0.0012 & 10 & $\operatorname{tr}$ & 89 & 11 & 100 & 0 & $\operatorname{tr}$ & red $\mathrm{Hb}, \mathrm{Bi}, \mathrm{Ep}, \mathrm{AKF}$ \\
\hline $6-4,115$ & 6.08 & 0.001 & 80 & 5 & 94 & 5 & 100 & 0 & 0 & $\mathrm{Q}, \mathrm{KF}, \mathrm{Bi}$ \\
\hline $6-5,86$ & 6.23 & 0.0005 & 25 & 0 & 80 & 15 & 85 & 0 & 15 & $\begin{array}{l}\text { KF, Bi, red } \mathrm{Hb}, \mathrm{Ap} \text {, } \\
\text { Ep, AKF, P, Z }\end{array}$ \\
\hline $6-6,134$ & 6.47 & 0.0004 & 30 & 2 & 83 & 15 & 100 & 0 & $\operatorname{tr}$ & $\mathrm{KF},(\mathrm{Z})$ \\
\hline $7-1,53$ & 6.61 & 0.0005 & 40 & $\operatorname{tr}$ & 90 & 10 & 100 & $\operatorname{tr}$ & $\operatorname{tr}$ & Q, Ep, AKF, Z \\
\hline $7-2,141$ & 7.11 & tr & 50 & 5 & 90 & 5 & 85 & 0 & 15 & $\mathrm{Bi}, \mathrm{Ep}, \mathrm{AKF}, \mathrm{P}$ \\
\hline $7-3,41$ & 7.22 & 0.0015 & 30 & 0 & 80 & 20 & 100 & 0 & $\operatorname{tr}$ & $\mathrm{P} *$ \\
\hline $7-4,59$ & 7.57 & tr & 30 & 5 & 90 & 3 & (63) & (0) & (37) & $\mathrm{Q}, \mathrm{Z}, \mathrm{AKF}, \mathrm{P}$ \\
\hline $7-5,20$ & 7.81 & 0.0005 & 90 & 0 & 85 & 15 & 67 & 0 & 33 & $\mathrm{Bi}, \mathrm{Ep}, \mathrm{Z}, \mathrm{AKF}$ \\
\hline $7-6,91 A$ & 8.28 & 0.2 & 60 & 0 & 60 & 40 & 30 & 0 & 70 & $\mathrm{Bi}^{*}, \mathrm{KF}, \mathrm{Ap}, \mathrm{P}$ \\
\hline $7-6,127$ & 8.35 & 0.002 & 80 & 0 & 75 & 25 & 100 & 0 & $\operatorname{tr}$ & red $\mathrm{Hb}, \mathrm{P}$ \\
\hline $8-1,127$ & 8.77 & $\operatorname{tr}$ & 50 & 2 & 89 & 9 & 73 & 0 & 27 & red $\mathrm{Hb}, \mathrm{Zr}, \mathrm{Ep}, \mathrm{AKF}, \mathrm{P}$ \\
\hline $8-2,48$ & 9.29 & $\operatorname{tr}$ & 50 & 5 & 84 & 10 & 90 & 0 & 10 & $\mathrm{Bi}, \mathrm{Ep}, \mathrm{P}^{*}, \mathrm{AKF}$ \\
\hline $8-3,77$ & 9.30 & 0.0025 & 90 & 0 & 83 & 17 & 77 & 0 & 23 & $\mathrm{Bi}^{*}, \mathrm{AKF}, \mathrm{P}^{*}$ \\
\hline $8-4,69$ & 9.60 & $\operatorname{tr}$ & 40 & 0 & 85 & 5 & 88 & 0 & 12 & $\mathrm{Q}^{*}$, red $\mathrm{Hb}, \mathrm{Bi}, \mathrm{Ep}, \mathrm{P}$ \\
\hline $8-5,23$ & 9.82 & 0.001 & 50 & 10 & 75 & 15 & 95 & 0 & 5 & $\mathrm{Bi}$, red $\mathrm{Hb}, \mathrm{Ep}, \mathrm{Z}, \mathrm{AKF}, \mathrm{P}$ \\
\hline $8-6,52$ & 10.20 & $\operatorname{tr}$ & 30 & 2 & 95 & 3 & (67) & (0) & (33) & $\mathrm{Q}, \mathrm{KF}, \mathrm{Bi}, \mathrm{Ep}, \mathrm{P}$ \\
\hline $9-1,136$ & 10.80 & $\operatorname{tr}$ & 30 & 0 & 100 & $\operatorname{tr}$ & (tr) & $(0)$ & $(0)$ & $\mathrm{Q}, \mathrm{P} *$ \\
\hline $9-2,109$ & 11.06 & $\operatorname{tr}$ & 40 & 1 & 94 & 5 & (70) & (0) & (30) & $\mathrm{Bi}, \mathrm{Q}, \mathrm{P}$ \\
\hline $9-3,22$ & 11.16 & 0.0012 & 60 & 1 & 68 & 30 & 100 & $\operatorname{tr}$ & tr & $\mathrm{KF}$, red $\mathrm{Hb}, \mathrm{Bi}, \mathrm{Ap}, \mathrm{AKF}, \mathrm{P}$ \\
\hline $9-4,60$ & 11.47 & $\operatorname{tr}$ & 10 & 2 & 93 & 5 & $(100)$ & $(0)$ & (0) & $\mathrm{Q}, \mathrm{P}$ \\
\hline $9-5,90$ & 11.76 & 0.0005 & 70 & 2 & 83 & 11 & 90 & 0 & 10 & $\mathrm{KF}, \mathrm{Ep}, \mathrm{Z}, \mathrm{P}, \mathrm{AKF}$ \\
\hline $10-1,105$ & 12.35 & 0.001 & 10 & 1 & 64 & 5 & (80) & (0) & (20) & $\mathrm{Bi}^{*}, \mathrm{KF}^{*}, \mathrm{AKF}, \mathrm{P}$ \\
\hline $10-2,113$ & 12.61 & 0.0003 & 40 & 5 & 70 & 9 & (66) & (22) & (12) & red $\mathrm{Hb}, \mathrm{KF}, \mathrm{Bi}^{*}, \mathrm{P}$ \\
\hline $11-2,129$ & 14.18 & 0.001 & 10 & 2 & 86 & 10 & 90 & 0 & 10 & $\operatorname{red} \mathrm{Hb}, \mathrm{Ep}, \mathrm{Z}, \mathrm{P}^{*}$ \\
\hline $11-4,39$ & 14.52 & 0.015 & 50 & $\operatorname{tr}$ & 96 & 4 & 42 & 0 & 58 & $\mathrm{Q}, \mathrm{Ep}, \mathrm{P}^{*}, \mathrm{Z}$ \\
\hline $11-6,60$ & 15.04 & $\operatorname{tr}$ & 20 & 5 & 50 & 5 & (80) & $(0)$ & (20) & $\mathrm{Bi}^{*}, \mathrm{AKF}, \mathrm{P}$ \\
\hline $12-248$ & 20.39 & 0.002 & 50 & 0 & 80 & 20 & 81 & 0 & 19 & $\mathrm{Bi}^{*}, \mathrm{KF}$, red $\mathrm{Hb}, \mathrm{AKF}^{*}, \mathrm{Z}$ \\
\hline $12-3,114$ & 20.49 & tr & 30 & $\operatorname{tr}$ & 90 & 5 & $(100)$ & $(0)$ & $(0)$ & $\mathrm{KF}, \mathrm{Z}, \mathrm{AKF}$ \\
\hline $12-5,72$ & 20.61 & tr & 60 & 5 & 85 & 10 & 100 & 0 & 0 & red $\mathrm{Hb}, \mathrm{Ep}, \mathrm{AKF}, \mathrm{Z}, \mathrm{P}$ \\
\hline $13-2,34$ & 20.82 & $\operatorname{tr}$ & 70 & 0 & 95 & $\operatorname{tr}$ & 21 & 0 & 79 & ?Garnet, Z \\
\hline $13-3,33$ & 20.89 & $\operatorname{tr}$ & 40 & 0 & 95 & 5 & $(100)$ & $(0)$ & $(0)$ & $\mathrm{Bi}$, red $\mathrm{Hb}, \mathrm{AKF}^{*}, \mathrm{KF}$ \\
\hline $13-5,141$ & 21.08 & $\operatorname{tr}$ & 10 & 10 & 70 & 10 & 100 & 0 & $\operatorname{tr}$ & $\mathrm{KF}, \mathrm{Z}$ \\
\hline $14-1,119$ & 21.23 & 0.0003 & 75 & 2 & 88 & 8 & (75) & $(25)$ & (0) & $\mathrm{Bi}^{*}, \mathrm{P}^{*}, \mathrm{KF}$ \\
\hline $14-3,23$ & 21.33 & 0.0012 & 80 & $\operatorname{tr}$ & 90 & 5 & 100 & 0 & 0 & $\mathrm{Bi}^{*}$, red $\mathrm{Hb}^{*}, \mathrm{AKF}, \mathrm{P}$ \\
\hline $14-4,71$ & 21.42 & 0.001 & 60 & 5 & 85 & 10 & 100 & 0 & $\operatorname{tr}$ & $\mathrm{Bi}$, red $\mathrm{Hb}, \mathrm{Z}, \mathrm{P}$ \\
\hline $14-6,66$ & 21.55 & $\operatorname{tr}$ & 50 & $\operatorname{tr}$ & 95 & 5 & $(67)$ & (0) & (33) & $\mathrm{Bi}, \mathrm{P}$ \\
\hline $15-1,135$ & 21.68 & 0.001 & 80 & $\operatorname{tr}$ & 93 & 7 & 79 & 0 & 21 & $\mathrm{Ep}, \mathrm{P}, \mathrm{Z}$ \\
\hline $15-3,60$ & 21.78 & $\operatorname{tr}$ & 70 & $\mathrm{tr}$ & 95 & 5 & 92 & 0 & 8 & $\mathrm{Bi}, \mathrm{Ep}, \mathrm{P}$ \\
\hline $15-5,146$ & 21.96 & 0.0005 & 50 & $\operatorname{tr}$ & 95 & 5 & 92 & 0 & 8 & red $\mathrm{Hb}, \mathrm{Z}$ \\
\hline $15-6,7$ & 21.96 & $\mathrm{tr}$ & 65 & 2 & 85 & 3 & (90) & (0) & (10) & $\mathrm{P}, \mathrm{AKF}^{*}$ \\
\hline
\end{tabular}


TABLE 1 - Continued

\begin{tabular}{|c|c|c|c|c|c|c|c|c|c|c|}
\hline $\begin{array}{l}\text { Sample (Top of } \\
\text { Interval in } \mathrm{cm} \text { ) }\end{array}$ & Age & Wt Res & $\begin{array}{l}\text { Volc } \\
(\%)\end{array}$ & $\begin{array}{l}\text { Glass } \\
(\%)\end{array}$ & $\begin{array}{l}\text { Plag } \\
(\%)\end{array}$ & $\begin{array}{l}\text { Maf } \\
(\%)\end{array}$ & $\mathrm{Hb}$ & Orpx & Clpx & Other \\
\hline $16-2,49$ & 22.15 & 0.02 & 60 & 5 & 78 & 12 & 93 & 0 & 7 & red $\mathrm{Hb}^{*}, \mathrm{KF}^{*}, \mathrm{Bi}^{*}, \mathrm{Ep}, \mathrm{P}$ \\
\hline $16-4,55$ & 22.29 & 0.017 & 50 & 30 & 65 & 5 & 42 & 31 & 27 & red $\mathrm{Hb}, \mathrm{Bi}, \mathrm{KF}, \mathrm{Z}$ \\
\hline $16-5,80$ & 22.37 & $\operatorname{tr}$ & 50 & 10 & 80 & 10 & 97 & 0 & 3 & red $\mathrm{Hb}, \mathrm{Bi}, \mathrm{KF}, \mathrm{Ep}, \mathrm{Z}$ \\
\hline $17-1,133$ & 22.55 & 0.06 & 75 & 80 & 15 & 5 & 7 & 0 & 93 & $\mathrm{Bi}^{*}$ \\
\hline $17-2,104$ & 22.61 & 0.108 & 90 & 70 & 25 & 5 & (12) & $(0)$ & (88) & $\mathrm{Bi}^{*}$ \\
\hline $17-4,144$ & 22.77 & 0.05 & 75 & 40 & 40 & 20 & 64 & 0 & 36 & $\mathrm{Bi}^{*}$, red $\mathrm{Hb}$ \\
\hline $17-5,46$ & 22.79 & 0.065 & 10 & 8 & 80 & 9 & 81 & 4 & 15 & $\mathrm{Bi}^{*}$, red $\mathrm{Hb}$ \\
\hline $18-1,143$ & 23.00 & 0.045 & 30 & 70 & 14 & 16 & 40 & 9 & 51 & $\mathrm{Bi}^{*}$, red $\mathrm{Hb}$ \\
\hline $18-5,89$ & 23.54 & 0.02 & 30 & 10 & 65 & 15 & 26 & 0 & 74 & $\mathrm{Bi}^{*}, \mathrm{KF}$ \\
\hline $19-2,37 \mathrm{~A}$ & 23.99 & 0.193 & 85 & 0 & 25 & 5 & 0 & 79 & 21 & \\
\hline $19-4,15$ & 24.27 & 0.075 & 95 & 70 & 22 & 8 & 43 & 6 & 51 & \\
\hline $19-6,78$ & 24.63 & 0.01 & 20 & 49 & 41 & 2 & 12 & 0 & 88 & $\mathrm{red} \mathrm{Hb}$ \\
\hline $20-2,105$ & 25.01 & 0.017 & 50 & 50 & 10 & $\operatorname{tr}$ & 13 & 19 & 68 & \\
\hline $21-2,73$ & 25.92 & 0.0055 & 40 & 65 & 38 & tr & (33) & (17) & $(50)$ & \\
\hline $21-4,116$ & 26.27 & 0.064 & 80 & 96 & 4 & $\mathrm{tr}$ & $(0)$ & (0) & $(100)$ & \\
\hline $22-2,57$ & 26.86 & 0.162 & 95 & 85 & 10 & 5 & 100 & 0 & 0 & red $\mathrm{Hb}$ \\
\hline $22-3 \quad 115$ & 27.07 & 0.085 & 70 & 0 & 4 & 1 & 0 & 19 & 81 & Bas. Glass \\
\hline $23-3,14$ & 27.91 & 0.04 & 2 & 0 & 0 & 0 & 0 & 0 & 0 & Bas. Glass \\
\hline $24-3,96$ & 28.95 & 0.0855 & 50 & 0 & 0 & 0 & 0 & 0 & 0 & Bas. Glass \\
\hline $25-2,109$ & 29.64 & 0.0185 & 2 & 10 & 20 & 0 & 0 & 0 & 0 & Bas. Glass \\
\hline $26-2,94$ & 30.13 & $(0.022)$ & 2 & 0 & 80 & 20 & (0) & (0) & $(100)$ & \\
\hline $27-1,92$ & 30.55 & L & tr & $\operatorname{tr}$ & 0 & 0 & 0 & 0 & 0 & \\
\hline $29-1,88$ & 31.55 & $\mathrm{~L}$ & $\operatorname{tr}$ & 0 & $\operatorname{tr}$ & 0 & 0 & 0 & 0 & \\
\hline $31-2,70$ & 32.62 & 0.0139 & $\operatorname{tr}$ & 0 & $\operatorname{tr}$ & 0 & 0 & 0 & 0 & \\
\hline $33-1,0$ & 33.50 & $(0.08)$ & 0 & 0 & 0 & 0 & 0 & 0 & 0 & \\
\hline $34-2,2$ & 35.57 & 0.0097 & 5 & 40 & 60 & 0 & 0 & 0 & 0 & $\mathrm{KF}, \mathrm{Q}$ \\
\hline $35-3,9$ & 37.64 & $\mathrm{~L}$ & 5 & 0 & 100 & 0 & 0 & 0 & 0 & $\mathrm{KF}$ \\
\hline $36-1,18$ & 38.35 & $\mathrm{~L}$ & (90) & (smear & r slide or & nly) & & & & $\begin{array}{l}\text { Riebeckite, authigenic } \\
\text { Glaucophane, KF, Clpx, } \\
\text { Plag, Glass }\end{array}$ \\
\hline $36-3,17$ & 38.58 & L & 5 & $\operatorname{tr}$ & 100 & 0 & 0 & 0 & 0 & $\mathrm{KF}$ \\
\hline $37-3,116$ & 39.36 & $\mathrm{~L}$ & 10 & 85 & 5 & 0 & 0 & 0 & 0 & \\
\hline $38-1,73$ & 39.82 & $\mathrm{~L}$ & 40 & 0 & 9 & 1 & 0 & 0 & $\operatorname{tr}$ & Bas. Glass \\
\hline $39-1,98$ & 40.55 & L & 20 & 0 & 100 & $\operatorname{tr}$ & $\operatorname{tr}$ & 0 & tr & \\
\hline \multicolumn{11}{|l|}{ Site 296} \\
\hline $1-1,94$ & 0.03 & 0.015 & 65 & 70 & 15 & 10 & 30 & 50 & 20 & \\
\hline $1-3,77$ & 0.11 & 0.012 & 95 & 50 & 28 & 2 & 27 & 42 & 31 & \\
\hline $2-2,131$ & 0.27 & 0.062 & 95 & 75 & 20 & 5 & 2 & 55 & 43 & $\mathrm{KF}, \mathrm{Bi}$ \\
\hline $2-3,20$ & 0.29 & 0.05 & 95 & 94 & 5 & $\operatorname{tr}$ & 33 & 50 & 17 & \\
\hline $2-3,120 \mathrm{~A}$ & 0.31 & 0.012 & 70 & 10 & 63 & 2 & 6 & 56 & 39 & \\
\hline $2-6,65$ & 0.43 & 0.291 & 95 & 90 & 9 & 1 & $\operatorname{tr}$ & 0 & 100 & \\
\hline $3-2,131$ & 0.55 & 0.6675 & 95 & 85 & 10 & 5 & 55 & 32 & 13 & $\mathrm{Bi}^{*}$, red $\mathrm{Hb}, \mathrm{P}$ \\
\hline $3-4,110$ & 0.64 & 0.07 & 85 & 15 & 10 & $\mathrm{tr}$ & 0 & tr & 0 & \\
\hline $4-1,124$ & 0.83 & 0.03 & 95 & 60 & 30 & 5 & 9 & 41 & 50 & \\
\hline $4-3,20$ & 0.89 & 0.006 & 70 & 20 & 58 & 2 & 10 & 40 & 50 & AKF \\
\hline $5-1,115$ & 1.06 & 0.005 & 60 & 70 & 25 & 2 & 18 & 39 & 43 & $\mathrm{Bi}$ \\
\hline $5-4,11$ & 1.16 & 0.07 & 60 & 70 & 20 & 5 & $(0)$ & (tr) & $(100)$ & $\mathrm{Bi}$ \\
\hline $6-2,12$ & 1.36 & 0.116 & 75 & 40 & 45 & 5 & 0 & 80 & 20 & $\mathrm{KF}$ \\
\hline $6-5,63$ & 1.50 & 0.0005 & 40 & 15 & 80 & $\mathrm{tr}$ & (0) & $(20)$ & $(80)$ & $\mathrm{Bi}, \mathrm{Q}^{*}, \mathrm{Z}, \mathrm{AKF}$ \\
\hline $7-3,143$ & 1.72 & 0.005 & 60 & 5 & 88 & 5 & 0 & 78 & 22 & \\
\hline $7-4,92$ & 1.75 & 0.0214 & 90 & 30 & 40 & 10 & 0 & 70 & 30 & \\
\hline $8-1,101$ & 1.90 & 0.02 & 50 & 60 & 30 & 5 & 0 & 80 & 20 & \\
\hline $8-3,61$ & 1.97 & 0.08 & 95 & 85 & 14 & 1 & 25 & 41 & 34 & $\mathrm{Ap}, \mathrm{KF}$ \\
\hline $8-3,123 \mathrm{~A}$ & 1.99 & 0.07 & 65 & 10 & 29 & 1 & 6 & 38 & 56 & $\mathrm{Z}$ \\
\hline $9-2,57$ & 2.21 & 0.02 & 60 & 50 & 30 & 10 & 15 & 38 & 47 & $\mathrm{AKF}$ \\
\hline $9-5,21$ & 2.33 & 0.025 & 50 & 50 & 40 & 10 & 88 & 0 & 12 & red $\mathrm{Hb}$ \\
\hline $10-2,102$ & 2.50 & 0.003 & 85 & 20 & 55 & 12 & 39 & 30 & 30 & \\
\hline $10-4,132$ & 2.60 & 0.04 & 10 & 65 & 35 & $\mathrm{tr}$ & (0) & (tr) & $(0)$ & \\
\hline $10-6,146$ & 2.69 & 0.0435 & 95 & 78 & 15 & $\operatorname{tr}$ & 69 & 27 & 3 & red $\mathrm{Hb}$ \\
\hline $11-1,145$ & 2.75 & 0.006 & 95 & 25 & 71 & 4 & 47 & 41 & 11 & $\mathrm{Bi}$ \\
\hline $11-4,33$ & 2.85 & 0.01 & 90 & 60 & 35 & 3 & 48 & 30 & 22 & $\mathrm{AKF}, \mathrm{Z}$ \\
\hline $12-1,98$ & 3.01 & 0.02 & 95 & 94 & 5 & 1 & 0 & 55 & 45 & $\mathrm{Z}$ \\
\hline $12-5,126$ & 3.20 & 0.008 & 90 & 25 & 50 & 10 & 60 & 20 & 20 & $\mathrm{KF}$ \\
\hline $13-1,95$ & 3.29 & 0.012 & 65 & 45 & 35 & 15 & 0 & 100 & 0 & \\
\hline $13-3,132$ & 3.39 & 0.017 & 90 & 50 & 46 & 1 & 26 & 56 & 18 & $\mathrm{Q}, \mathrm{AKF}$ \\
\hline $14-2,138$ & 3.64 & 0.065 & 95 & 95 & 4 & $\operatorname{tr}$ & (100) & $(0)$ & (0) & \\
\hline $14-3,51$ & 3.66 & 0.02 & 95 & 38 & 30 & 10 & 44 & 36 & 20 & red $\mathrm{Hb}, \mathrm{KF}$ \\
\hline $14-6,87$ & 3.81 & 0.02 & 30 & 5 & 70 & 25 & 32 & 48 & 20 & \\
\hline
\end{tabular}


TABLE 1 - Continued

\begin{tabular}{|c|c|c|c|c|c|c|c|c|c|c|}
\hline $\begin{array}{l}\text { Sample (Top of } \\
\text { Interval in } \mathrm{cm} \text { ) }\end{array}$ & Age & Wt Res & $\begin{array}{l}\text { Volc } \\
(\%)\end{array}$ & $\begin{array}{l}\text { Glass } \\
(\%)\end{array}$ & $\begin{array}{l}\text { Plag } \\
(\%)\end{array}$ & $\begin{array}{r}\text { Maf } \\
(\%)\end{array}$ & $\mathrm{Hb}$ & Orpx & Clpx & Other \\
\hline $15-2,103$ & 3.90 & 0.085 & 95 & 90 & 8 & 1 & 20 & 60 & 20 & $\mathrm{Zr}, \mathrm{Z}$ \\
\hline $15-3,32 \mathrm{~A}$ & 3.92 & 0.765 & 100 & 90 & 7 & 0 & 0 & 0 & 0 & \\
\hline $15-3,79$ & 3.94 & 0.255 & 95 & 95 & 5 & 0 & 0 & 0 & 0 & \\
\hline $16-1,122$ & 4.14 & 0.017 & 95 & 60 & 28 & 4 & 20 & 53 & 27 & red $\mathrm{Hb}$ \\
\hline $16-4,124$ & 4.27 & 0.008 & 40 & 35 & 50 & 15 & 23 & 35 & 41 & $\mathrm{KF}$ \\
\hline $16-6,52$ & 4.34 & 0.10 & 95 & 1 & 22 & 2 & 0 & 66 & 34 & $\mathrm{Q}, \mathrm{AKF}, \mathrm{Z}$ \\
\hline $17-3,136$ & 4.51 & 0.009 & 95 & 4 & 92 & 2 & 44 & 44 & 12 & $\mathrm{z}$ \\
\hline $17-5,71$ & 4.58 & 0.02 & 50 & 20 & 70 & 10 & 40 & 40 & 20 & \\
\hline $18-1,58$ & 4.68 & 0.001 & 80 & $\mathrm{tr}$ & 95 & 4 & 50 & 50 & $\operatorname{tr}$ & red $\mathrm{Hb}$ \\
\hline $18-2,98$ & 4.73 & 0.007 & 50 & 1 & 96 & 3 & 80 & 0 & 20 & \\
\hline $19-2,60$ & 5.02 & 0.042 & 75 & 40 & 50 & 10 & 64 & 24 & 12 & $\mathrm{Bi}^{*}$ \\
\hline $19-4,94$ & 5.65 & 0.025 & 95 & 68 & 20 & 10 & 61 & 24 & 15 & $\mathrm{Bi}^{*}$ \\
\hline $20-1,146$ & 6.50 & 0.001 & 50 & 0 & 75 & 24 & 100 & 0 & 0 & red $\mathrm{Hb}, \mathrm{KF}$ \\
\hline $20-2,75$ & 6.63 & 0.0002 & 40 & $\operatorname{tr}$ & 99 & 1 & $(100)$ & (0) & (0) & $\mathrm{Z}, \mathrm{AKF}$ \\
\hline $20-3,105$ & 6.93 & 0.0005 & 50 & 15 & 80 & 3 & (67) & (0) & (33) & red $\mathrm{Hb}$ \\
\hline $20-4,95$ & 7.17 & $\mathrm{tr}$ & 15 & 5 & 95 & tr & (70) & (0) & (30) & $\mathrm{Bi}, \mathrm{AKF}$ \\
\hline $21-1,100$ & 8.00 & $(0.01)$ & 1 & 30 & 70 & 0 & 0 & 0 & 0 & \\
\hline $21-2,46$ & 8.17 & 0.002 & 60 & 1 & 95 & 4 & 91 & 9 & 0 & red $\mathrm{Hb}, \mathrm{Bi}, \mathrm{AKF}$ \\
\hline $21-3,92$ & 8.48 & 0.055 & 95 & 70 & 18 & 2 & 6 & 39 & 56 & \\
\hline $21-4-102$ & 8.67 & 0.045 & 95 & 94 & 2 & 1 & (14) & (29) & (57) & \\
\hline $21-5,133$ & 9.05 & 0.017 & 90 & 10 & 70 & $\operatorname{tr}$ & (0) & (57) & (43) & \\
\hline $21-6,94$ & 9.23 & 0.02 & 90 & 40 & 19 & 1 & 22 & 28 & 50 & red $\mathrm{Hb}, \mathrm{Z}$ \\
\hline $22-1,141$ & 9.65 & 0.002 & 75 & 0 & 49 & 1 & (70) & (0) & (30) & red $\mathrm{Hb}^{*}, \mathrm{Z}$ \\
\hline $22-2,38$ & 9.73 & 0.005 & 15 & 40 & 40 & 20 & 60 & 0 & 40 & $\mathrm{Bi}$ \\
\hline $22-3,17$ & 9.95 & 0.001 & 40 & 75 & 24 & 1 & $(0)$ & (0) & $(100)$ & $\mathrm{Bi}, \mathrm{AKF}$ \\
\hline $22-4,9$ & 10.10 & 0.0035 & 50 & 75 & 24 & $\operatorname{tr}$ & (0) & (30) & (70) & \\
\hline $22-5,138$ & 10.44 & 0.0002 & 20 & 1 & 95 & 4 & $(100)$ & (0) & (0) & red $\mathrm{Hb}$ \\
\hline $22-6,29$ & 10.49 & $\operatorname{tr}$ & 75 & 5 & 90 & 5 & 50 & 7 & 43 & $\mathrm{Bi}$ \\
\hline $23-1,133$ & 10.83 & 0.027 & 5 & 80 & 20 & 0 & 0 & 0 & 0 & \\
\hline $23-2,118$ & 10.99 & $(0.03)$ & 50 & 80 & 17 & 1 & $(0)$ & (20) & (80) & \\
\hline $23-3,69$ & 11.11 & 0.0025 & 90 & 75 & 23 & 2 & 100 & $\mathrm{tr}$ & 0 & $\mathrm{Bi}$, red $\mathrm{Hb}$ \\
\hline $24-1,41$ & 11.82 & 0.0015 & 90 & 70 & 20 & 6 & 50 & 33 & 17 & $\mathrm{Bi}$ \\
\hline $24-2,110$ & 12.07 & 0.012 & 70 & 80 & 17 & 3 & 36 & 36 & 28 & $\mathrm{Q}, \mathrm{Bi}, \mathrm{AKF}$ \\
\hline $24-3,91$ & 12.22 & 0.002 & 40 & 40 & 48 & 11 & 30 & 30 & 40 & \\
\hline $24-4,32$ & 12.40 & 0.002 & 50 & 30 & 65 & 4 & 55 & 14 & 31 & \\
\hline $24-6,130$ & 12.78 & 0.003 & 80 & 60 & 25 & 5 & 81 & 2 & 17 & \\
\hline $25-1,89$ & 12.96 & 0.015 & 80 & 75 & 20 & 5 & 100 & tr & 0 & $\mathrm{Bi}, \mathrm{KF}$ \\
\hline $25-2,102$ & 13.14 & 0.017 & 95 & 65 & 20 & 8 & 42 & 25 & 33 & $\mathrm{KF}$ \\
\hline $25-3,114$ & 13.33 & 0.04 & 90 & 45 & 40 & 10 & 46 & 27 & 27 & \\
\hline $25-4,111$ & 13.50 & 0.031 & 85 & 78 & 15 & 5 & 0 & 80 & 20 & \\
\hline $26-1,130$ & 16.02 & 0.03 & 60 & 68 & 25 & 5 & 28 & 21 & 51 & \\
\hline $26-2,67$ & 16.04 & 0.051 & 50 & 50 & 40 & 10 & 30 & 50 & 20 & \\
\hline $26-4,141$ & 16.15 & 0.017 & 60 & 15 & 80 & 5 & 22 & 46 & 32 & Ap \\
\hline $27-2,124$ & 16.32 & 0.018 & 80 & 10 & 85 & 4 & 4 & 50 & 46 & $\mathrm{Bi}, \mathrm{Z}, \mathrm{AKF}$ \\
\hline $27-3,85$ & 16.35 & 0.047 & 80 & 5 & 20 & 5 & 5 & 24 & 71 & $\mathrm{Z}$ \\
\hline $28-2,84$ & 16.56 & 0.175 & 90 & 90 & 9 & $\mathrm{tr}$ & (0) & (33) & (67) & $\mathrm{Z}$ \\
\hline $28-3,42 \mathrm{~A}$ & 16.59 & 0.872 & 100 & 98 & 1 & $\mathrm{tr}$ & (0) & $(\mathrm{tr})$ & (tr) & \\
\hline $28-3,96$ & 16.61 & 0.301 & 40 & 96 & 4 & $\operatorname{tr}$ & (0) & (100) & (0) & KF \\
\hline $28-4,120 \mathrm{~A}$ & 16.65 & 0.1625 & 98 & 95 & 5 & tr & (tr) & $(0)$ & $(\mathrm{tr})$ & $\mathrm{Bi}^{*}, \mathrm{Zr}$ \\
\hline $28-422$ & 16.63 & L & 30 & 60 & 40 & $\mathrm{tr}$ & $(0)$ & (tr) & $(0)$ & \\
\hline $28-5,98$ & 16.69 & 0.06 & 85 & 90 & 8 & $\mathrm{tr}$ & 55 & 27 & 18 & \\
\hline $29-1,13$ & 16.76 & 0.06 & 85 & 90 & 8 & 1 & 29 & 29 & 42 & $\mathrm{Bi}$ \\
\hline $29-3,20$ & 16.84 & 0.316 & 95 & 95 & 9 & $\operatorname{tr}$ & (0) & $(\mathrm{tr})$ & (tr) & \\
\hline $29-5,36$ & 16.92 & 0.03 & 40 & 40 & 28 & 1 & 0 & 9 & 91 & \\
\hline
\end{tabular}

Note: $\mathrm{A}=$ labeled volcanic ash; Age = estimated age in m.y., taken from recorded depth and depth vs. age curves by linear interpolation; Wt. Res $=$ weight in $\mathrm{g}$ of residue $(325$ mesh $=43 \mu)$ from 2 -cc sample; $\mathrm{L}=$ lithified, parentheses for poorly estimated weight due to lithification; \% Volc $=$ percent of volcanic material in aliquot of total residue; $\mathrm{tr}=$ trace amount; $\%$ Blass = percent of clear glass in total volcanic material of aliquot; $\%$ Plag = percent of plagioclase in total volcanic material of aliquot; $\% \mathrm{Maf}=$ percent of mafic minerals in total volcanic material of aliquot; $\mathrm{Hb}$, Orpx, and $\mathrm{Clpx}=$ percent of brown or green hornblende, orthopyroxene, and clinopyroxene, respectively, in total of these three minerals, parentheses indicate less than 10 grains counted total; Other: $\mathrm{Q}=$ quartz (volcanic or terrigenous), $\mathrm{KF}=\mathrm{K}$ feldspar, (generally volcanic or terrigenous, but perhaps including some authigenic); $\mathrm{AKF}=$ authigenic $\mathrm{K}$-feldspar; $\mathrm{Bi}=$ biotite; red $\mathrm{Hb}=$ red hornblende; $\mathrm{Ap}=$ apatite $\mathrm{Zr}=$ zircon; $\mathrm{Ep}=$ epidote; $\mathrm{Z}=$ zeolite (indet.) $\mathrm{P}=$ phillipsite; Bas. Glass = clear basaltic glass (brown, low positive relief). Asterisks indicate an exceptional amount. 
TABLE 2

Constituents Distinguished From Samples at Sites 293, 294, and 295

\begin{tabular}{|c|c|c|c|c|c|c|c|}
\hline $\begin{array}{l}\text { Sample (Top of } \\
\text { Interval in } \mathrm{cm} \text { ) }\end{array}$ & (\%) Volc & (\%) Glass & (\%) Plag & $\mathrm{Hb}$ & Orpx & Clpx & Other \\
\hline \multicolumn{8}{|l|}{ Site 293} \\
\hline $2-2,140$ & 80 & 0 & 70 & 50 & 0 & 50 & $\mathrm{KF}$ \\
\hline $2-5,27$ & 50 & 6 & 90 & 100 & 0 & $\operatorname{tr}$ & \\
\hline $2-5,47$ & 70 & 1 & 60 & (70) & $(30)$ & $\operatorname{tr}$ & \\
\hline $2-5,59$ & 80 & 3 & 70 & 67 & tr & 33 & $\operatorname{red} \mathrm{Hb}, \mathrm{Bi}, \mathrm{KF}, \mathrm{Z}$ \\
\hline $2-5,64$ & 95 & 0 & 83 & 0 & 80 & 20 & \\
\hline $4-2,81$ & 95 & $\operatorname{tr}$ & 65 & 67 & $\operatorname{tr}$ & 33 & $\mathrm{Ap}$, red $\mathrm{Hb}$ \\
\hline $5-4,133$ & 40 & 0 & 100 & $\operatorname{tr}$ & 0 & 0 & Wood, KF \\
\hline $6-2,25.5$ & 60 & $\mathrm{tr}$ & 95 & 38 & 13 & 63 & red $\mathrm{Hb}$ \\
\hline $6-2,100$ & 50 & $?$ & 50 & 0 & 0 & $(100)$ & \\
\hline $8-2,77$ & 80 & 0 & 100 & 25 & 75 & $\operatorname{tr}$ & \\
\hline $10-2,69$ & 30 & 0 & 30 & 0 & 0 & 0 & \\
\hline $15-1,95$ & 95 & 0 & 94 & 95 & 0 & 5 & $\mathrm{Bi}, \mathrm{KF}$, red $\mathrm{Hb}$ \\
\hline $15-1,101$ & 40 & 0 & 50 & 10 & 0 & 90 & $\mathrm{Z}, \mathrm{AKF}$ \\
\hline $15-1,130$ & 40 & 0 & 80 & $\operatorname{tr}$ & 0 & $\operatorname{tr}$ & FD \\
\hline $16-3,79$ & 70 & 0 & 20 & 58 & 8 & 34 & $\mathrm{KF}$, red $\mathrm{Hb}, \mathrm{FD}$ \\
\hline $17-3,81$ & 50 & 0 & 60 & 0 & 0 & 0 & $\mathrm{Ap} *, \mathrm{Bi}$ \\
\hline $18-1,62$ & tr & 0 & $\operatorname{tr}$ & $\operatorname{tr}$ & 0 & 0 & FD \\
\hline \multicolumn{8}{|l|}{ Site 294} \\
\hline $1-4,70$ & 85 & 0 & 70 & 50 & 0 & 50 & \\
\hline $3-5,87$ & $\operatorname{tr}$ & 0 & $\operatorname{tr}$ & 0 & 0 & 0 & unident. opaque \\
\hline $4-3,78$ & 5 & 0 & 100 & $\operatorname{tr}$ & 0 & 0 & FD, auth Q? \\
\hline $4-4,146$ & 5 & 0 & 100 & $\operatorname{tr}$ & 0 & 0 & $\mathrm{Ap}, \mathrm{FD}, \mathrm{MN}$ \\
\hline $6-1,62$ & 0 & 0 & 0 & 0 & 0 & 0 & $\mathrm{MN}, \mathrm{FD}$ \\
\hline \multicolumn{8}{|l|}{ Site 295} \\
\hline $1-5,73$ & 50 & 0 & 80 & 0 & 0 & $\operatorname{tr}$ & $\mathrm{Q}^{*}, \mathrm{FD}, \mathrm{AKF}$ \\
\hline $2-2,42$ & 60 & 0 & 60 & 0 & 0 & 0 & $\mathrm{Mt}, \mathrm{FD}$ \\
\hline $2-6,106$ & 20 & 0 & 100 & 0 & 0 & 0 & FD \\
\hline $3-4,35$ & 2 & 0 & 100 & 0 & 0 & 0 & $\mathrm{Q}, \mathrm{FD}$ \\
\hline
\end{tabular}

Note: Same as for Table 1, except that Wt Res, \%MAF, and Age are omitted. These samples have not been treated with acid and represent a coarser fraction $(62 \mu$ rather than $43 \mu)$ than those reported in Table 1 . One sample $(293-15-1,130 \mathrm{~cm})$ has abundant carbonate-coated grains of volcanic crystals, comparable grains would not have been seen in the Site 292 and 296 samples. Additional symbols: $\mathrm{FD}=$ fish debris; $\mathrm{Mt}=$ magnetite $; \mathrm{MN}=$ micronodules .

\section{PETROGRAPHY}

The volcanic debris from Sites 292 and 296 is fairly homogeneous in character. It generally consists of wellsorted, highly angular, fine debris, suggesting dispersal as wind-borne rather than as pumice-rafted debris. Glass is generally dominant in fresher samples, with plagioclase most abundant in altered samples. Variations in the relative amounts of clear glass, plagioclase, and mafic minerals are apparently independent of later alteration. Some variation evidently results from a relatively high proportion of glass in the samples with the highest proportion of total volcanics. Still other variations are not explained, such as the occasional high-mafic content. High-biotite and other mineralogically peculiar samples evidently reflect variations in magmatic chemistry.

Coarser crystalline constituents, especially plagioclase and clinopyroxene, are characteristically shattered with conchoidal fractures, evidently reflecting highly explosive eruptive activity. Hornblende is generally cleaved, and the smaller grains tend to be euhedral, or subhedral as a result of magmatic resorption. A preserved glass investment on many of these small grains (pyroxenes, hornblende, quartz) shows that this rounding does not result from diagenesis.

\section{Clear Glass}

Clear glass is generally the dominant form of glass. It is rarely entirely absent, even when authigenic minerals are abundant. The glass exists as bubble, or tubular pumice. Some of the bubble glass evidently had relatively large diameter vesicles; relics consist of nearly flat, angular plates. Altered glass is commonly more equant than fresh glass, and angular, almost polyhedral blocks are common. Bubble glass is commonly more abundant than tubular pumice.

\section{Porphyritic Glass}

Porphyritic, andesitic glass is generally subordinate, but widely occurring. It varies towards the clear form, less commonly, to the brown form. It is generally highly altered, and some was probably misidentified as nonvolcanic authigenic mineral aggregates. In doubtful cases, the presence of plagioclase microlites facilitates identification. Aside from plagioclase, one sample (296-16-6, $52 \mathrm{~cm}$ ) contains orthopyroxene microphenocrysts. 


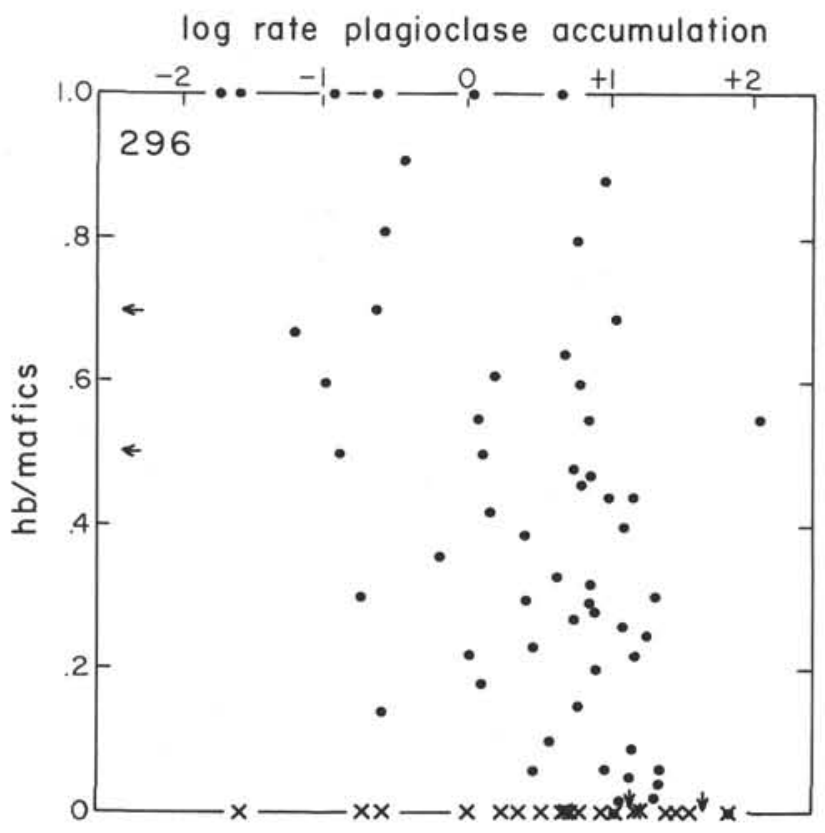

Figure 5. Curve showing for Site 296 the relationship between the proportion of brown and green hormblende to total mafics (sum hornblende + clinopyroxene + orthopyroxene) against the log of accumulation rate of volcanic plagioclase (g/cm 2 m.y.). Arrows indicate trace amounts of sediments; " $x$ " symbols the absence of hornblende.

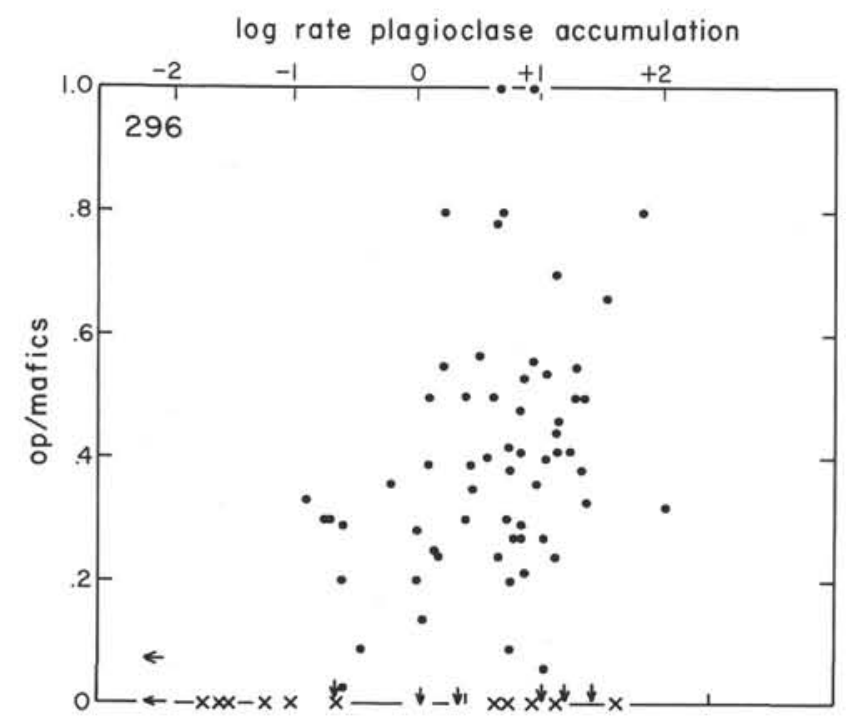

Figure 6. Curve showing for Site 296 the relationship between the proportion of orthopyroxene to total mafics (as in Figure 5) against the log of accumulation rate of volcanic plagioclase $\left(\mathrm{g} / \mathrm{cm}^{2} \mathrm{~m} . \mathrm{y}\right.$.).
At Site 292, porphyritic glass is scarce in the upper part (Cores 1 to 8 ) with only one sample in Core 3 having large amounts. In the lower part (Cores 19 to 22), this glass is more conspicuous. At Site 296, it is far more common than at Site 292 being the predominant volcanic constituent in Cores 3, 8, 16, and 22 .

\section{Brown Glass}

Fresh brown glass has a refractive index similar to Caedex and is probably basaltic. It is generally nonporphyritic, or contains unrecognizably small microlites. It is of bubble form, evidently representing scoria. This glass is in most cases scarce, but conspicuous at Site 292 in Cores 1, 3, 10 (where it is palagonitized in 292-101, $105 \mathrm{~cm}$ ), Cores 23-25, and in Core 27 from Site 296.

\section{Plagioclase}

Plagioclase is the most ubiquitous of the volcanic mineral species. Although generally anhedral (conchoidally or otherwise fractured irregularly), a few grains are euhedral. Most grains are compositionally zoned, and show simple twinning. Polysynthetic twinning is relatively rare, and these grains (Site 292) may be sedimentary detritus. The plagioclase has a variable, but commonly low refractive index. Possibly, much of the plagioclase has reacted with pore waters to generate more sodic rim.

The recognition of plagioclase, many grains of which are untwinned, requires some experience. It is based on the visible compositional zoning, and a distinctive dispersion which gives characteristic brown and purple extinction colors. Much plagioclase also contains twophase fluid or glass inclusions.

\section{K-feldspar: Volcanic and Authigenic}

$\mathrm{K}$-feldspar is generally a scarce constituent. Although it was more conspicuous at Site 292 , there was no particular pattern of occurrence of this mineral at either site.

Volcanic K-feldspar is water clear, commonly simply twinned, showing some optical zoning and dispersion. The very low refractive index and low negative $2 \mathrm{~V}$ are also distinctive.

Authigenic $\mathrm{K}$-feldspar is rarely as euhedral as had been found in Caribbean sediments (Donnelly and Nalli, 1973). Some grains are commonly crowded with inclusions, often arranged more or less parallel to the Caxis. Some authigenic $\mathrm{K}$-feldspar occurs as formless, multicrystalline aggregates, recognized by their birefringence, relief, and texture. Authigenic K-feldspar occurs in samples from Sites 292 and 296, but it is especially common in that part of Site 292 where glass is virtually absent.

\section{Quartz}

Quartz grains of purely volcanic origin are scarce. All of the four occurrences from Site 296 are presumed to be volcanic, as well as the occurrence in Core 1 from Site 292 where quartz grains are invested with glass. The scattered quartz occurrences below Core 2 at Site 292 are considered to represent sedimentary dispersal rather 
than a volcanic origin. These occurrences are suspiciously parallel with occurrences of epidote and polysynthetically twinned plagioclase, all of which are considered to originate in a low-grade metamorphic environment. In samples of Core 292-13 a lone grain of pink garnet was also found. Characteristic tiny fluid inclusions (which are all of one phase) are present in the presumably metamorphic quartz.

\section{Zeolites: Phillipsite}

The only zeolite positively identified (optically or by X-ray diffraction) was phillipsite. At Site 292 the appearance of phillipsite correlates with the disappearance of glass, providing strong circumstantial evidence for a volcanic origin. At Site 296, phillipsite was only positively identified in one sample, but several other occurrences of "zeolite" at the same site are probably phillipsite.

Most of the phillipsite is in the form of characteristic angularly terminated prisms. In grain residues, however, most zeolite occurs in odd multicrystalline lumps showing numerous small crystalline faces and reentrants.

\section{Epidote}

This mineral occurs in Cores 3 through 16 from Site 292. It is rarely common, but easily recognized. Most grains are of one crystal only, but a few are multicrystalline. Epidote is presumed here to be an indicator of transport by turbidity current from a metamorphic source. The limited abundance of epidote (and cooccurring presumably metamorphic quartz) indicates that most of the crystalline debris at this site represents volcanic activity.

\section{Hornblende}

Hornblende is the second most widespread mineral species in these sediments. There is no positive evidence for metamorphic amphibole (with the epidote-quartz suite at Site 292). Most hornblende occurs in euhedral (cleaved?) or subhedral rounded grains. The commonest colors are shades of brown to olive to green, but bright red hornblende also occurs throughout in small quantities.

Some distinctively colored varieties probably merit further work: very pale-colored (292-11-6, 60; 296-21-2, 46; and 296-24-4, 32); conspicuous golden varieties (29213, 296-5, 296-20; and 296-24); hornblendes with a high optical absorption, possibly representing a more alkalic magmatic origin, (292-16-2, 49 and 292-16-5, 80); a deep brown hornblende (292-18-1, 143); and a hornblende, pleochroic in pink and green, (296-24-2, 110; 296-22-1, 141; 296-23-3, 69; and 292-6-2, 35).

A most distinctive euhedral deep-green riebeckite (length fast, $n x$ about 1.69) was seen in an alkalic ash from Site 292 (292-36-1, 18), occurring with a euhedral clinopyroxene, glass, K-feldspar, plagioclase, and authigenic glaucophane.

\section{Glaucophane}

The most unusual mineral species found was glaucophane, which occurred as tiny prisms in Sample
292-36-1, 18. The euhedral crystal form, and occasional intergrowth with evidently authigenic K-feldspar implies that it is an authigenic product of the alteration of an especially alkalic ash which produced augite, riebeckite, and $\mathrm{K}$-feldspar. The glaucophane is deeply colored, distinctively pleochroic, but evidently concentrically zoned on a fine scale. The slow refractive index of about 1.63, implies a low iron content. However, these grains are length fast, characteristic of crossite.

The designation of an authigenic nature for glaucophane is based on its occurrence as rather long, thin prisms, some of which are aggregated in a radial pattern, and occasional intergrowth with authigenic (?) feldspar. Another heretofore low-grade metamorphic mineral, spessartine, was found as an evident authigenic mineral in Leg 15 sediments (Donnelly and Nalli, 1973).

\section{Biotite}

Biotite is minor but widespread, especially at Site 292. It is invariably brown, and occasionally shows a hexagonal subhedral crystal form. It often has polygonal holes; the hexagonal ones were probably occupied by apatite, but other holes appear to have been occupied by a monoclinic mineral.

The biotite of Site 292 shows a pattern of alteration parallel to, but not identical to, that of glass, and the appearance of phillipsite. Cores 1 and 2 have altered biotite, Cores 3 through 10 have mainly altered biotite, with fresh grains in Cores $3,4,7,8$, and 10 . Cores 14 through 18 have only fresh material.

\section{Apatite}

Apatite is far less common in the western Pacific than it had been in older volcanic material from the Caribbean (Donnelly, 1973). The occurrences are noted in Tables 1 and 2 .

\section{Clinopyroxene}

In all probability all of the clinopyroxene found at both sites is augite. It is invariably pale green, rarely with a distinct yellowish tinge. Particularly pale grains were found in 296-16, and especially deeply colored green grains were seen in Cores 1, 3, 17, and 18 of Site 292 and Cores 21 and 22 from Site 296.

\section{Orthopyrexene}

Orthopyroxene (all of which is probably hypersthene) is less common than clinopyroxene. Most grains are rounded or deeply etched. The etch pattern resembles that of clinopyroxene, but has an orthorhombic symmetry.

The distinctive pink-blue green pleochroism of orthopyroxene helps to identify this mineral, which never shows the nearly universal pale green color of clinopyroxene. However, much orthopyroxene is very pale in color. Site 292, Cores 1, 2, and 19 had some pale orthopyroxene, and Cores 3 through 7 and 17 had mostly the pale variety. At Site 296 pale orthopyroxene was found in Cores 1, 2, 5, 10, 16, 19, 22, 24, 27, and 28. 


\section{Zircon}

Zircon was a scarce volcanic mineral, being principally associated with biotite. The biotite-rich ash from 296$28-4,120 \mathrm{~cm}$ had crystals of zircon $10 \mu$ wide, but zircons in Samples 292-5-5, 24, 292-8-1, 127, and 296-15-2, 103 were much finer grained.

\section{PATTERNS OF ERUPTIVE ACTIVITY}

Table 1, and Figures 7 and 8, document the fact that the mineralogical character of ash depends somewhat on the amount of ash, i.e., the eruptivity shown by the sample. Thus, the relative amount of clear glass is highest in those samples which represent the largest eruptions. The more crystalline samples also occur during waning periods of eruption. Further, the relative amount of mafic minerals is lowest in the most abundant ash samples, and highest in samples with a limited ash content.

A further mineralogical distinction is worth noting. Orthopyroxene is a relatively abundant mafic mineral in ashes representing large eruptions (Figure 8), but hornblende is relatively more abundant during periods of limited activity (Figure 7). The two minerals show an antithetical relationship with eruptive activity, expressed as rate of accumulation of volcanic plagioclase.

The glass/crystal ratio and the orthopyroxene/ hornblende ratio probably reflect the same phenomena. High eruptivity may result from bringing a very hot, almost completely liquid melt to the surface. A more crystallized melt has already lost much of its heat and, thus, potential explosive power. Because feldspars crystallize first and in the greatest abundance, and hornblende probably replaces orthopyroxene through magmatic reaction, the observed relationships are consistent with a model of variable extent of intratelluric crystalli- zation. Further substantiation for the reaction of orthopyroxene in the melt is the observed rounding of grains completely invested with glass.

\section{VOLCANIC HISTORY OF LUZON: SITE 292}

The Tertiary tectonic evolution of Luzon is summarized by Gervasio (1973). Oligocene strata, aside from possible unfossiliferous early Oligocene beds, are absent, so historical inferences drawn from stratigraphy for this period are essentially impossible. This time period is represented by granitic stocks emplaced during an orogenic event. The early Miocene had "intermittent, but markedly reduced basaltic and andesitic effusions," followed by quartz diorite plutons in the middle Miocene. The late Miocene was a time of "thick and extensive dacitic tuffs and tuffaceous clastics, in places with associated andesitic and dacitic flows and agglomerates. . The highly eruptive and siliceous volcanic activity extended to the early Pliocene." A later PlioPleistocene event formed "volcanic piedmont deposits around volcanic cones," and the extrusions of plateau basalts.

The present analysis (Figure 5) shows that the volcanic activity of Luzon in the Oligocene was moderate, rising to a maximum at the Oligocene-Miocene boundary. It fell off in the early Miocene, with the accumulation rate decreasing, and the number of samples with minimal ("trace") amounts of volcanic material increasing.

A hiatus recorded at Site 292 is followed by middle Miocene sediments showing moderate activity. This activity diminishes, but increases to another poorly defined maximum in the late Miocene (12 to 7 m.y.), with sporadic eruptions. At about 5 m.y. the activity increases, reaching a maximum during the Recent which is

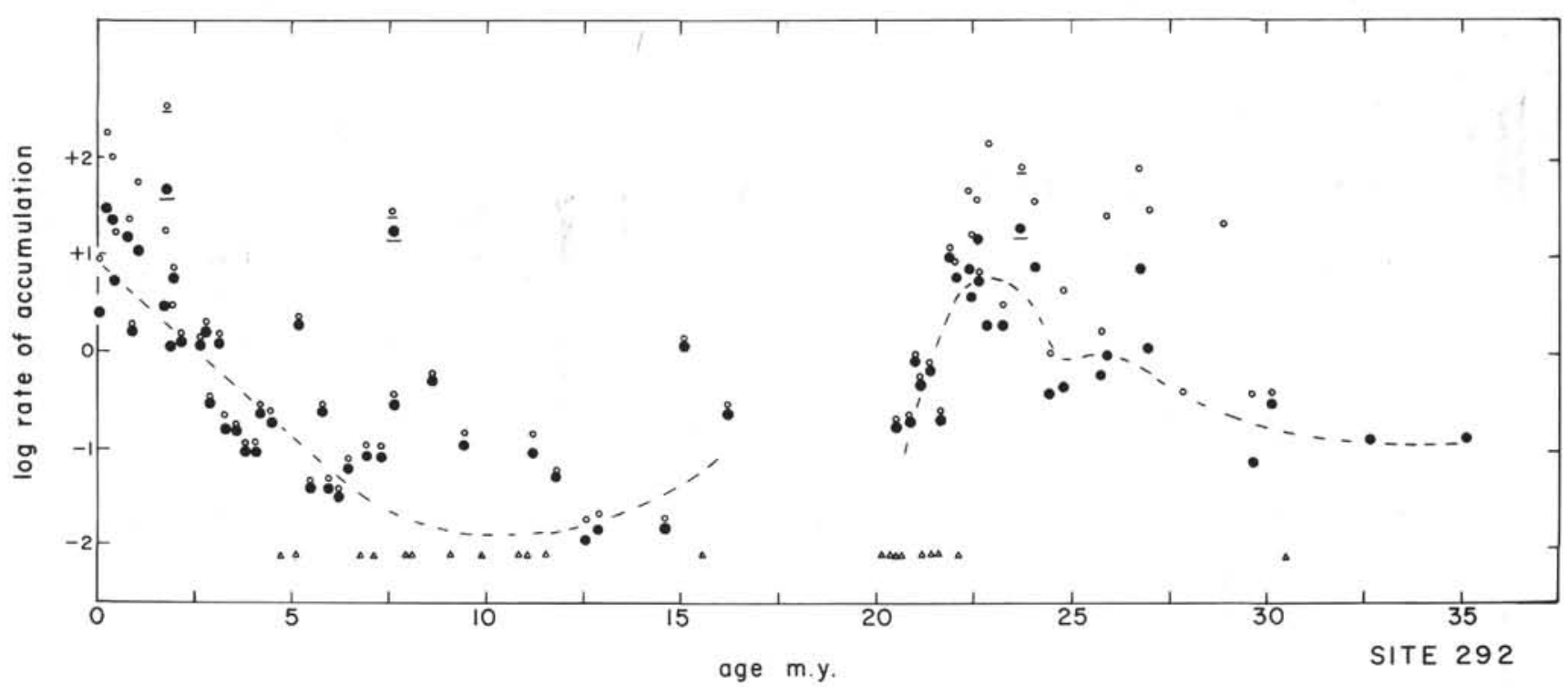

Figure 7. Plot showing for Site 292 the log of the accumulation rate for total volcanic debris (open circles) and plagioclase (solid circles) $\left(\mathrm{g} / \mathrm{cm}^{2} \mathrm{~m} . y\right.$.), against age of sediment. Dashed line shows trend for plagioclase. Tri-angles indicate samples with unmeasurably small quantities of volcanic debris. Underlined symbols are for samples labeled "ash." 


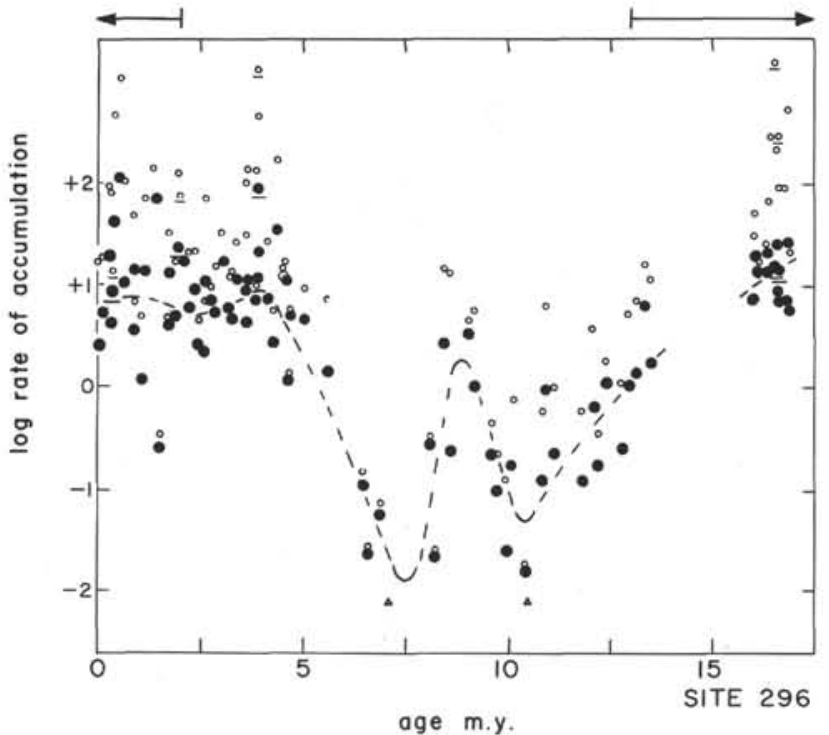

Figure 8. Plot showing for Site 296 the relationship between log accumulation rate versus age of sediment, as in Figure 7. Arrows at top show times of Neogene volcanic activity in central Japan (Sugimuro, personal communication).

quantitatively and qualitatively similar to that of about 22 m.y. ago.

The occurrence of quartz and epidote in the 22 m.y. to about $1.5 \mathrm{~m} . y$. interval implies the transport of metamorphic debris from Luzon during that time by turbidity currents. The present bathymetry implies that such debris must have been carried a considerable distance uphill. This is a formidable difficulty not diminished by the inference that the paleobathymetry was similar.

In the Caribbean, turbidites from South America were traced to Sites 150 and 31 of DSDP Legs 15 and 4, respectively (Donnelly, 1973); again paleobathymetry would appear to require uphill transport, possibly as much as 1000 meters in the case of Site 31. The mechanism is not clear. Internal derivation of the debris from the crust of the Benham Rise is possible; the mineral assemblage is not definitive for either continental or oceanic crustal origin, although it does imply a low grade of metamorphism. Derivation of the debris from Luzon, however, requires that this derivation cease in the Pleistocene with the growth of the Philippine Trench and its northward extension, which became an efficient sediment trap for debris from Luzon.

\section{VOLCANIC HISTORY OF SOUTHERN JAPAN: SITE 296}

Debris on the Palau-Kyushu Ridge is presumed to come principally from southwestern Japan (Kyushu, southwestern Honshu) and the Ryukyu Islands; some component may be derived from the central Japan-Izu Islands volcanic trend (Figure 1). This latter derivation would require transport of coarse debris in a southward or westward direction, in opposition to the eastwardmoving high-altitude winds. There seems to be no adequate mineralogical criterion for a separation of two source belts, and there is no hint in the present work that multiple volcanic provenance areas may be involved.

Sugimura and Uyeda (1973) discuss the geologic development of western Japan primarily by analogy with better-known east Japan provinces. In southwestern Japan, volcanic activity was considered to be more important in the pre-Neogene, although some spectacular volcanic centers, such as Aso, remain to the Recent. Sugimura and Uyeda note that tholeiitic magmatic activity of the "northern zone" (Sea of Japan side of southwestern Honshu) of the early Miocene gave way to subalkalic activity in the middle and late Miocene of the median zone (of southwestern Honshu). From a variety of evidence they conclude, " ...the sinking rate of the ocean lithosphere underthrusting the southwest Honshu Arc decreased from the early Miocene to the middle Miocene. A maximum in the middle Miocene was followed by the peak of activity in the late Miocene. The whole activity seems to have ended in the early Pleistocene Epoch."

In eastern Japan, Sugimura and Uyeda conclude that the volume of early Neogene (about 25 to 22 m.y.) volcanic material was about $50,000 \mathrm{~km}^{3} / \mathrm{m} . y$., about $1000 \mathrm{~km}^{3} / \mathrm{m} . \mathrm{y}$. during the mid-late Neogene (22 to 2 m.y.), increasing to $2500 \mathrm{~km}^{3} / \mathrm{m} . \mathrm{y}$. in the last $2 \mathrm{~m} . \mathrm{y}$. Sugimura (personal communication) has recently extended the younger boundary of the early Neogene to 13 m.y.

The present study (Figure 6) largely confirms the twostage (early Neogene and Quaternary) groupings of Sugimura and Uyeda, but adds some important information. The early Neogene volcanic activity declined to a minimum value in the middle Miocene (about 11 m.y.), and increased sharply to a short-lived peak about 9 m.y. It declined again in the late Miocene and increased sharply at the Miocene-Pliocene boundary. The late period of intense activity from $5 \mathrm{~m}$.y. to the present might be divisible into an early and late more active period and a middle, less volcanic, period.

\section{CONCLUSIONS}

The deep-sea volcanic ash study presented here substantiates the previous work of Sugimura and Uyeda (1973), but refines their results. The striking rise of volcanic activity at 5 m.y. correlates at Sites 292 and 296 , though the rise is far more dramatic at Site 296. Unpublished data from Central America (DSDP Site 83) and the results from the Lesser Antilles (Donnelly, 1973) further suggest a very widespread, perhaps worldwide, change in volcanic activity of subduction zones. The implied change in spreading rates of the world ocean is not as apparent. However, a recent study in the northeast Pacific (Blakley, 1974) shows that for the 17 m.y. to 9 m.y. period, the inferred spreading rate decreased gradually by about one-half to about $10 \mathrm{~m} . \mathrm{y}$. and rose abruptly to the earlier high value at $9 \mathrm{~m}$.y. It may be coincidental that the volcanic activity curve for Site 296 is precisely parallel to this, but Site 292 shows only a poor parallel trend.

The results presented herein appear to provide an insight into a new question of global tectonics: is sea-floor spreading directly related to calcalkaline volcanic activity, does it vary with time, and on a worldwide basis? 


\section{ACKNOWLEDGMENTS}

Sample preparation work for the present study was financed by a grant from the University Awards Committee of the State University of New York, and by further help from the Center for Solid Earth Geology of the State University of New York at Binghamton. Able assistance was provided by Bruce Gaither, Mark Griswold, and Roy Wilkens, to whom I am very grateful.

I am further grateful to Arata Sugimura for numerous stimulating discussions on the subject of Japanese volcanic history during his visit to this department, as well as for more recent correspondence on the subject.

\section{REFERENCES}

Blakley, R.J., 1974. Fine-scale variation in spreading rates in the northeast Pacific (abstract): EOS Am. Geophys. Union Trans., v. 55 , p. 299.

Donnelly, T.W., 1973. Circum-Caribbean explosive volcanic activity: evidence from Leg 15 sediments. In Edgar, N.T., Saunders, J.B., et al., Initial Reports of the Deep Sea Drill- ing Project, Volume 15: Washington (U.S. Government Printing Office), p. 966-988.

Donnelly, T.W. and Nalli, G., 1973. Mineralogy and chemistry of Caribbean sediments. In Edgar, N.T., Saunders, J.B., et al., Initial Reports of the Deep Sea Drilling Project, Volume 15: Washington (U.S. Government Printing Office), p. 929-962.

Gervasio, F.C. 1973. Geotectonic development of the Philippines. In Coleman, P.J. (Ed.), The Western Pacific: Island Arcs, Marginal seas. Geochemistry: (Univ. of Western Australia Press), p. 307-324.

Kuno, H., 1962. Catalogue of the active volcanoes and solfatara fields of Japan, Taiwan, and Marianas: Internat. Volcanological Assoc. (Catalogue of the Active Volcanoes of the World), Part 11, $332 \mathrm{p}$.

Neumann van Padang, M., 1953. Catalogue of the active volcanoes and Solfatara fields of the Philippine Islands and Cochin China: Internat. Volcanological Assoc. (Catalogue of the Active Volcanoes of the World), Part 2, 49 p.

Sugimura, A. and Uyeda, S., 1973. Island Arcs: Japan and its environs: New York (Elsevier). 

PLATE 1

Typical glass textures (scale bar is $100 \mu \mathrm{m}$ long)

Figure 1 Bubble glass; late Oligocene; 292-22-2, $57 \mathrm{~cm}$.

Figure 2 Tubular pumice; platy glass; Pleistocene; 296-2-6, $65 \mathrm{~cm}$.

Figure 3 Platy glass; large hornblende crystal; Pleistocene; 296-3-2, $131 \mathrm{~cm}$.

Figure 4 Platy glass; etched clinopyroxene crystal; Pliocene; 296-12-1, $98 \mathrm{~cm}$.

Figure 5 Tubular pumice; bubble glass, showing color variation; middle Miocene; 296-28-3, $96 \mathrm{~cm}$.

Figure 6 Bubble glass, biotite crystal; middle Miocene; 296$28-4,120 \mathrm{~cm}$ 
PLATE 1
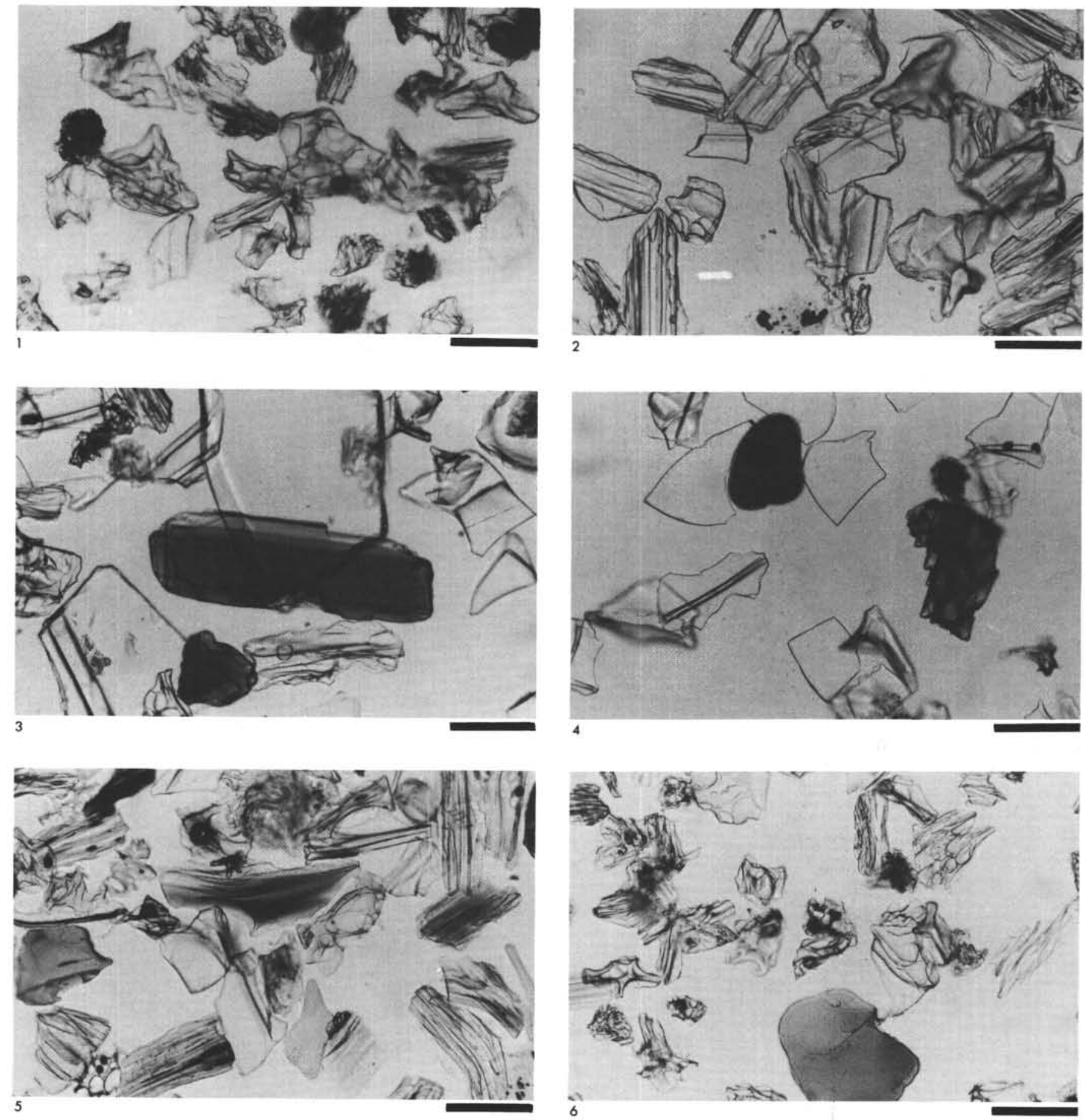


\section{PLATE 2}

Volcanic crystals and glass textures (scale bar is $100 \mu \mathrm{m}$ long)

Figure 1 Magmatically rounded hornblende crystal invested with glass; bubble glass; Pleistocene; 292-1$4,7 \mathrm{~cm}$.

Figure 2 Degraded glass remnant; Pleistocene; 292-2-2, 66 $\mathrm{cm}$.

Figure 3 Hornblende crystals, showing range in colors from pale to highly absorbent; Pliocene; $292-4-1,91 \mathrm{~cm}$.

Figure 4 Degraded glass remnant (center); plagioclase; hornblende; late Miocene; 292-5-6, $60 \mathrm{~cm}$.

Figure $5 \quad$ Biotite crystal (top center); etched clinopyroxene crystal (top right); plagioclase crystal (right center); middle Miocene; 292-8-3, $77 \mathrm{~cm}$.

Figure 6 Unetched orthopyroxene crystal; bubble glass; Pleistocene; 296-1-3, $77 \mathrm{~cm}$.

Figure 7 Porphyritic glass; middle Miocene; 296-22-1, 141 $\mathrm{cm}$.

Figure $8 \quad$ Bubble glass, showing color variation; early Miocene; 296-28-2, $84 \mathrm{~cm}$. 


\section{PLATE 2}
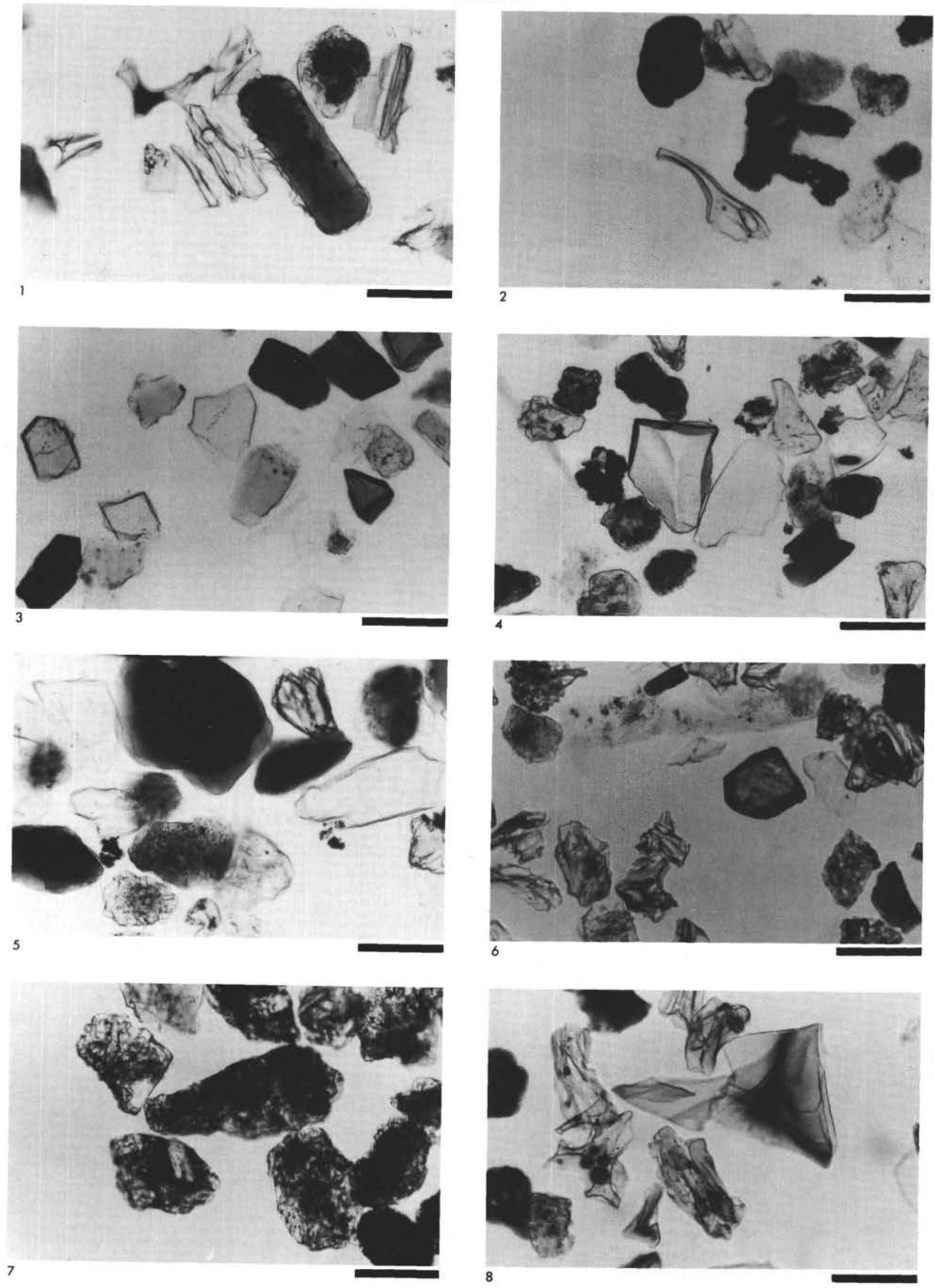


\section{PLATE 3}

Authigenic minerals; volcanic crystals (scale bar is $100 \mu \mathrm{m}$ long)

Figure $1 \quad$ Magmatically rounded clinopyroxene invested with glass (right center); brown (basaltic) glass (left center); Pleistocene; 292-1-1, $74 \mathrm{~cm}$.

Figure 2 Riebeckite euhedron; glass fragments; late Eocene; 292-36-1, $18 \mathrm{~cm}$.

Figure 3 Authigenic glaucophane crystal (center); late Eocene; 292-36-1, $18 \mathrm{~cm}$.

Figure 4 Unetched clinopyroxene euhedron; glass fragments; late Eocene; 292-36-1, $18 \mathrm{~cm}$.

Figure $5 \quad$ Authigenic K-feldspar crystal; late Pliocene; 296$11-4,33 \mathrm{~cm}$.

Figure $6 \quad$ Authigenic K-feldspar euhedron; early Miocene; 296-26-5, $102 \mathrm{~cm}$. 
PLATE 3
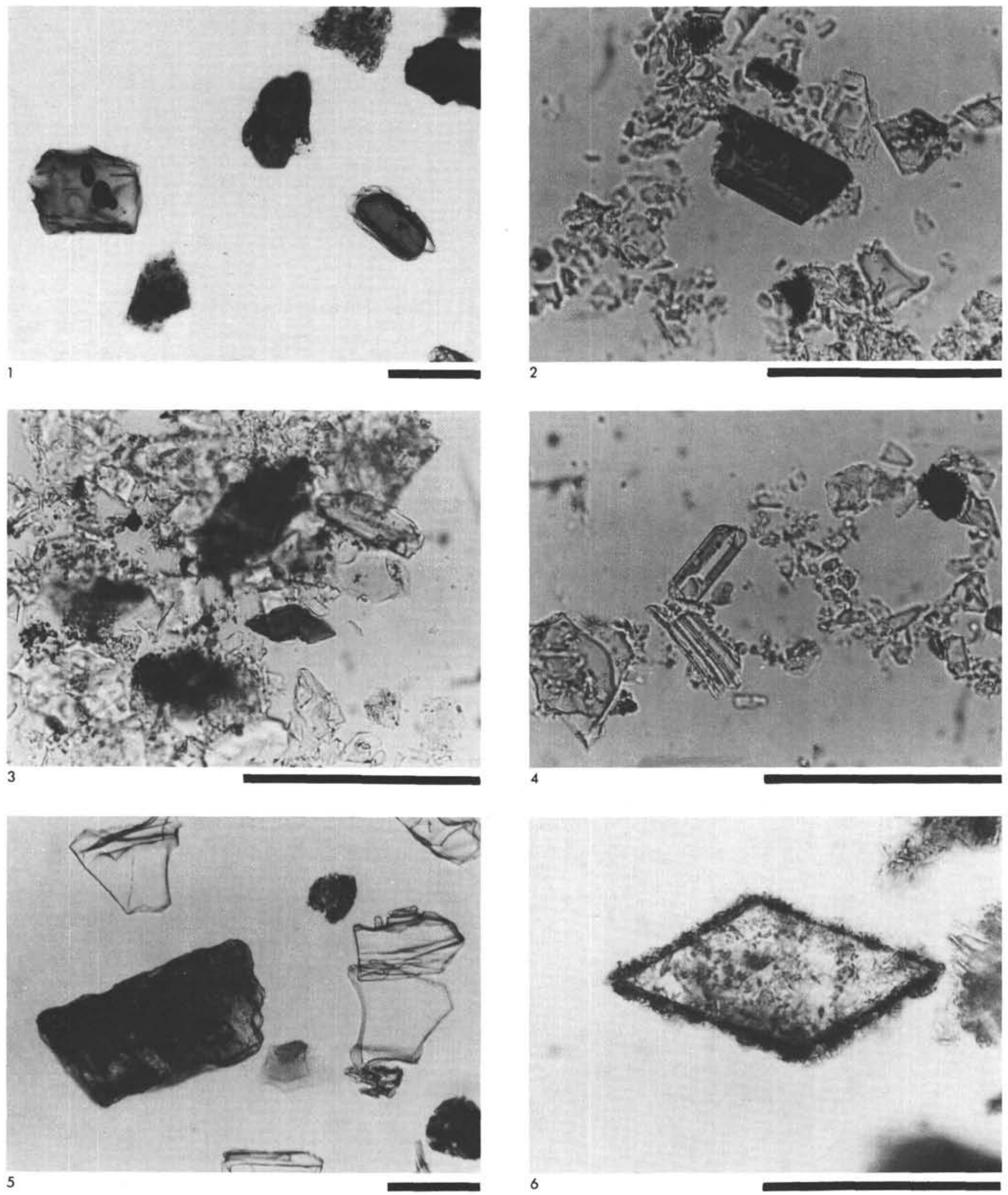


\section{PLATE 4}

Volcanic crystals; zeolite: glass (scale bar is $100 \mu \mathrm{m}$ long)

Figure 1 Fractured (right) and euhedral, glass invested clinopyroxene (left) crystals; Pleistocene; 292-1-1, $74 \mathrm{~cm}$.

Figure 2 Etched clinopyroxene; hornblende crystals; late Pliocene; 292-3-4, $30 \mathrm{~cm}$.

Figure 3 Etched orthopyroxene; late Oligocene; 296-21-5, $133 \mathrm{~cm}$.

Figure 4 Etched clinopyroxene; late Oligocene; 296-21-5, $133 \mathrm{~cm}$.

Figure 5 Etched orthopyroxene; late Pliocene; 296-11-4, 33 $\mathrm{cm}$.

Figure 6 Brown (basaltic) glass and clear glass, showing relief contrast; Pleistocene; 296-5-1, $115 \mathrm{~cm}$.

Figure 7 Zeolite "lump" (center, highest relief); clinopyroxene fragment (right center); glass fragments; late Pliocene; 296-8-3, $123 \mathrm{~cm}$.

Figure $8 \quad$ Zeolite "lump" (right, highest relief); clinopyroxene (left center); plagioclase; early Miocene; 296$27-2,124 \mathrm{~cm}$. 
PLATE 4
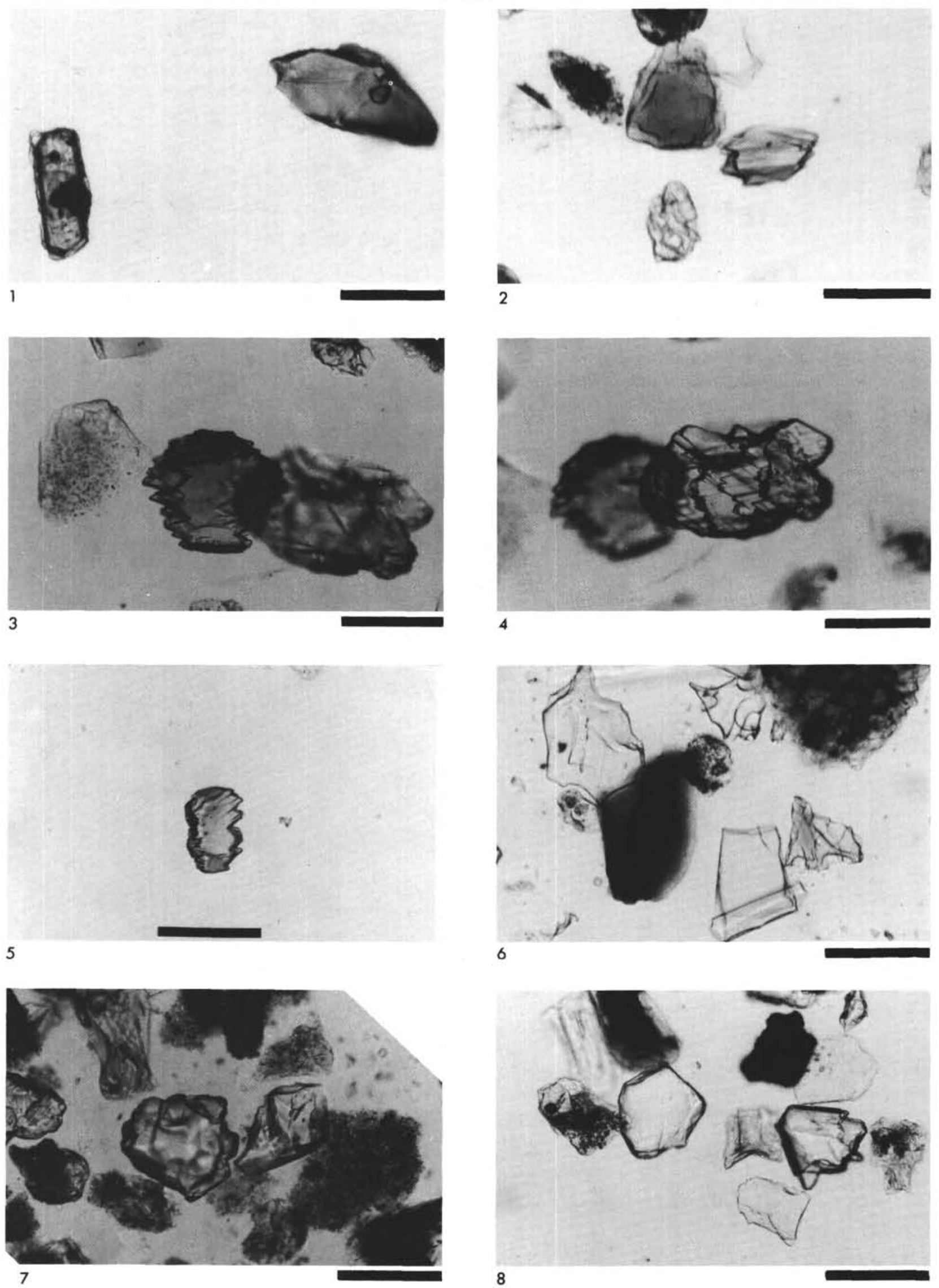